\title{
Quantitative Assessment of Uncertainties and Sensitivities in the Estimation of Life Loss Due to the Instantaneous Break of a Hypothetical Dam in Switzerland
}

\author{
Anna Kalinina, Matteo Spada *(D) and Peter Burgherr (D) \\ Laboratory for Energy Systems Analysis, Paul Scherrer Institute, Forschungsstrasse 111, 5232 Villigen PSI, \\ Switzerland; kalinina.anna@outlook.com (A.K.); peter.burgherr@psi.ch (P.B.) \\ * Correspondence: matteo.spada@psi.ch
}

Citation: Kalinina, A.; Spada, M.; Burgherr, P. Quantitative Assessment of Uncertainties and Sensitivities in the Estimation of Life Loss Due to the Instantaneous Break of a Hypothetical Dam in Switzerland. Water 2021, 13, 3414. https: / / doi.org/10.3390/w13233414

Academic Editor: Paolo Mignosa

Received: 4 October 2021

Accepted: 25 November 2021

Published: 2 December 2021

Publisher's Note: MDPI stays neutral with regard to jurisdictional claims in published maps and institutional affiliations.

Copyright: (c) 2021 by the authors. Licensee MDPI, Basel, Switzerland. This article is an open access article distributed under the terms and conditions of the Creative Commons Attribution (CC BY) license (https:// creativecommons.org/licenses/by/ $4.0 /)$.

\begin{abstract}
High safety standards of operators and regulators for dams in Switzerland require periodic assessments of risk mitigation measures at dams. Therefore, risk assessments need to include the estimation of life loss (LL) due to a potential dam break. This study demonstrated the benefits of applying the HEC-LIFESim software for modelling LL due to the instantaneous break of a hypothetical dam in Switzerland. HEC-LIFESim overcomes limitations of empirical methods by modelling evacuation and warning processes. Furthermore, for credible LL estimates, metamodelling was used to quantify uncertainty in model parameters. Polynomial chaos expansion (PCE) was applied to approximate the LL model of HEC-LIFESim using only 550 runs. Uncertainty in the model inputs was propagated through the metamodel to quantify uncertainty in the LL estimates. Finally, a global sensitivity analysis was performed by calculating Sobol' and Borgonovo indices. The results demonstrate that the three-parameter population in a locality within all considered localities, fatality rate in the chance zone, and warning issuance delay contributed most to the variability of the LL estimates. The application of the proposed methodology can support risk management by providing detailed and accurate risk measures and helping in prioritizing safety measures to be considered and implemented.
\end{abstract}

Keywords: dam break; global sensitivity analysis; life loss; polynomial chaos expansion; risk assessment; uncertainty quantification

\section{Introduction}

The estimation of life loss (LL) from a potential dam failure is an important part of dam risk analysis [1]. LL estimates can be applied as a risk measure to assess if the potential risk posed by the dam exceeds the acceptable limits set by authorities [2]. Furthermore, LL estimates can be used to identify dam failure scenarios and factors that may cause severe consequences and, with this knowledge, support risk-based decision-making procedures for dam safety purposes [3]. Finally, LL estimates can help to evaluate a group of dams within a portfolio and identify those dams that need the most attention, e.g., structural modifications [4].

Dam risk analysis methods must provide credible LL estimates; however, most of the available methods for LL estimation are regression-based equations [5,6]. Derived from empirical data of historical observations, these equations possess a number of limitations: they do not differentiate between characteristics of the dam failure and flood severity, information on many parameters represents averages, etc. [7]. In recent years, fuzzy set theory has been applied to introduce information such as characteristics of the dam failure, flood severity, cause of the failure, population distribution, etc. [8-10]. However, as with regression-based equations, these studies come with a series of limitations, for example, the lack of physical interactions between receptors (e.g., people and flood wave). To overcome the limitations of the abovementioned methods, models simulating physical processes 
and interactions between physical receptors taking place during a flood event need to be applied [11]. In this context, the most established and used models are the Life Safety Model (LSM) $[12,13]$ and the HEC-LIFESim model $[14,15]$. While both models are agent-based models, they differ in their focus. LSM is more suited for modelling the behaviour of an individual receptor (i.e., a person or a car during evacuation) since it simulates each person, building, and vehicle in a floodplain and their interaction with the flood wave based on fundamental physical equations. In contrast, HEC-LIFESim scales up the simulation to a mesoscale by simulating the re-distribution of people in response to an emergency warning through the implementation of a traffic simulation model which interacts directly with the flood wave propagation. Therefore, HEC-LIFESim is more reasonable in the context of dam risk assessment since it gives a more general overview of the LL estimates in the area downstream of a dam. Based on these premises, HEC-LIFESim was applied in this study. The software uses information about evacuation, warning, structures, and routes to reflect site-specific conditions. Hence, a limited availability of data for these parameters can constrain the application of this software to specific dam locations. In the case of Switzerland, detailed digital maps and databases available for the entire country area $[16,17]$ enable the application of HEC-LIFESim for dam risk analysis reflecting Swiss conditions.

In recent years, there has been growing acceptance that LL estimates in the models of dam failures are uncertain [18]. An important contribution to the uncertainty is due to factors (e.g., hydrological parameters [19]) affecting the LL estimates, which are intrinsically uncertain [20]. Thus, point LL estimates cannot fully reflect the potential range of the consequences due to a dam failure. Therefore, the consideration of uncertainty is important in dam failure LL estimation.

Dynamic spatial models estimating LL commonly use a Monte Carlo analysis to account for uncertainties in the physical characteristics [13,21,22]. In particular, the Uncertainty Mode of HEC-LIFESim propagates uncertainties associated with model parameters through the model to provide probability distributions of LL estimates [14,23]. A Latin hypercube sampling technique is used for the generation of values for model input parameters; however, this approach has certain limitations. First, the scarcity of available data can make a rigorous description of the uncertainty in input parameters challenging [24]. Another limitation is a rather simplified representation of the parameter probabilities due to a specific research focus, for example, on the extreme values of the parameters and not on the entire domain. Due to data scarcity and such vague representation of the parameter probabilities, a large number of model runs can be required to achieve convergence of the mean or to achieve the desired confidence (e.g., a user wants to be $95 \%$ certain that the model prediction is within $1 \%$ of the mean) [25]. A large number of model runs is often in strong contrast with the advanced and complex simulation models encountered in engineering applications [26], which is a similar problem to the one proposed in this study.

To overcome the aforementioned limitations, a framework for quantitative assessment of uncertainties based on metamodelling introduced by Sudret [27] and De Rocquigny et al. [28] was applied. To construct a metamodel, the HEC-LIFESim model was adjusted to Swiss conditions and approximated with a polynomial chaos expansion (PCE) using only a limited number of runs of the original model [29]. The uncertainty of the model inputs, described with the probability distributions reflecting the entire range of their uncertainty, was propagated through the metamodel, allowing quantification of the uncertainty of the LL estimates with much lower computational costs with respect to Monte Carlo based approaches.

In addition to uncertainty analysis, sensitivity analysis (SA) has been suggested by the developers of HEC-LIFESim as a tool that enables understanding the contribution of uncertain model inputs to the variability of the model output [30,31]. Furthermore, SA helps achieve more robust results and confidence about key model inputs [32] but also prioritize safety measures based on the most relevant parameters affecting the results [33,34]. In general, there are two types of sensitivity analysis: local (LSA) and global (GSA) [35]. Analyses 
conducted in the dam safety risk assessment studies are examples of the former, where only one parameter is changed at a time and only for relatively small increments $[15,36]$. The limitation of LSA is that it does not allow variation of multiple parameters at a time and the input parameters are not sampled over the entire possible space of their uncertainty. Therefore, GSA methods such as Sobol' indices and Borgonovo indices are applied in this study to overcome these limitations [37-40]. These methods also allow identification of the non-influential inputs to fix them to deterministic values, calibration of model inputs, and mapping the output behaviour as a function of the inputs [41].

Based on these premises, the goal of the current study was threefold. First, the application of the HEC-LIFESim software for LL estimation in the failure of a hypothetical hydropower dam in Switzerland was demonstrated. This application can encourage the use of dynamic spatial models for emergency management within the Swiss dam safety field. Second, uncertainties in the constructed LL model were quantitatively assessed using the PCE-based method. In addition to lower computational cost, the constructed PCE metamodel allows the representation of conditions relevant for Switzerland by considering a population of inhabited localities downstream of large hydropower dams as it was described by Kalinina, Spada, Vetsch, Marelli, Whealton, Burgherr, and Sudret [34]. Third, a GSA was performed to evaluate model inputs that are influential for a potential decrease of LL consequences in the event of a dam failure. Application of several GSA methods allowed for quality assurance of the conclusions drawn.

The rest of the paper is structured as follows. In Section 2, the computational model used for the LL estimation is introduced, including all information sources and assumptions. Then, methods used for quantification of uncertainties and sensitivities of the model inputs and outputs are explained in Section 3. The analysed scenarios are also defined there. Results for the probability distributions of the model inputs and outputs as well as all calculated sensitivity indices are given in Section 4. Finally, Section 5 provides a discussion of the results, and conclusions are provided.

\section{Computational Model}

A dam-break model commonly consists of (i) the simulation of the outflow hydrograph resulting from the dam break, (ii) the propagation of this hydrograph in the downstream topography, and (iii) the LL estimation. The first two steps were addressed previously by Kalinina, Spada, Vetsch, Marelli, Whealton, Burgherr, and Sudret [34], while this study addressed the LL estimation.

For the first two steps of the dam-break model, a concrete arch dam over $100 \mathrm{~m}$ in height was chosen, which reflects typical Swiss conditions [34]. Furthermore, a complete and instantaneous failure was assumed for this dam [42]. That model predicted flow quantities at the end of the downstream valley and estimated their uncertainty.

With the same assumptions and using the computed flow quantities as input, the LL model was generated in this study using HEC-LIFESim [14]. Developed by the Hydrologic Engineering Center (HEC) of the U.S. Army Corps of Engineers, HEC-LIFESim uses by default input data provided by different American agencies; however, to be able to realistically simulate a scenario for Switzerland, input data need to be specific for local conditions.

The remainder of this section contains information about the HEC-LIFESim software and its modules. Furthermore, it describes sources for the input data used in the LL model for a Swiss case.

\subsection{The HEC-LIFESim Software}

HEC-LIFESim is a spatial dynamic simulation software for estimating the consequences of a flood event (e.g., LL). It considers processes that affect LL through its three modules: (1) loss of shelter, (2) warning and evacuation, and (3) life loss (Figure 1). These modules exchange data through GIS layers such as ESRI maps [43] and other datasets described in the following subsections. 


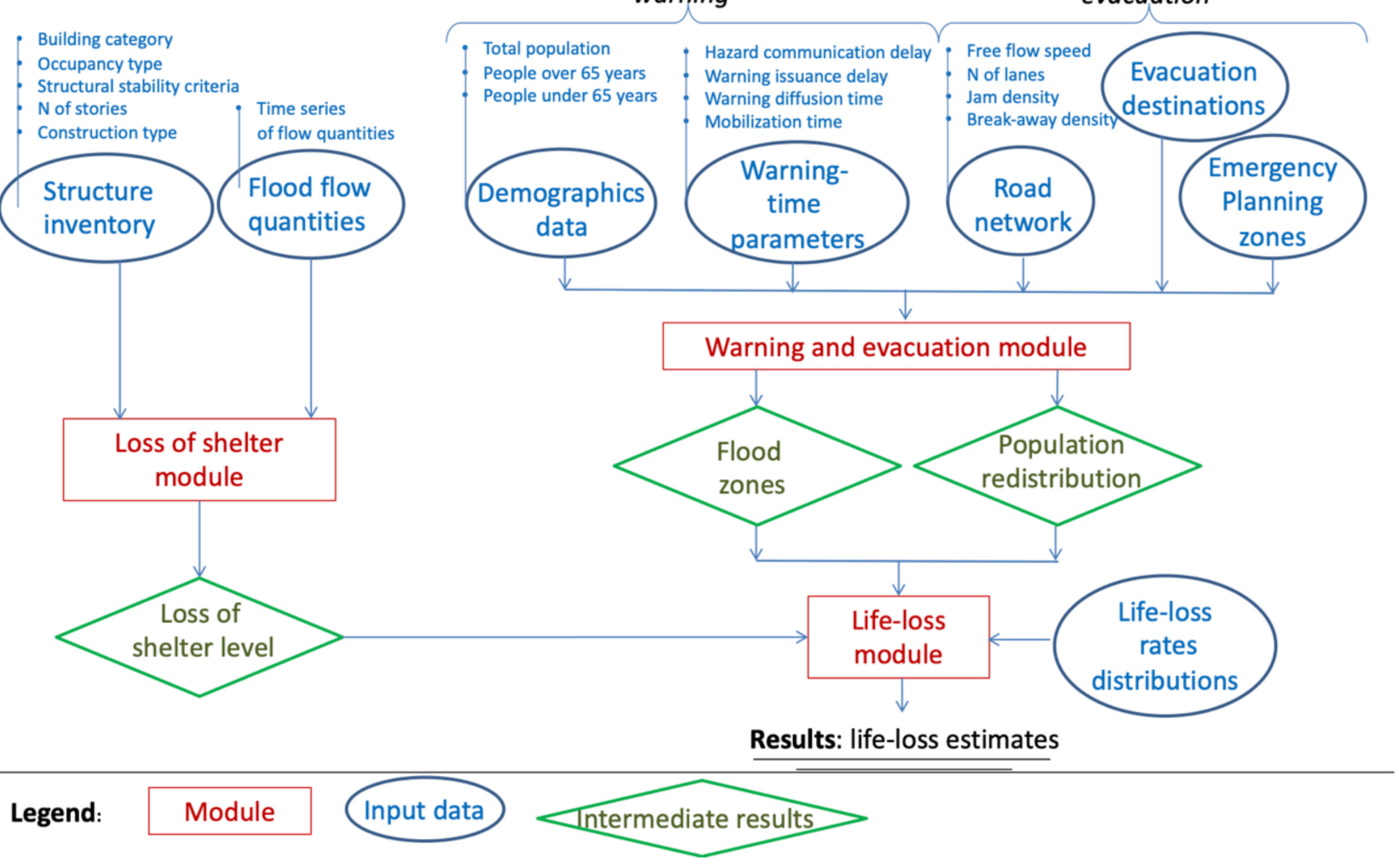

Figure 1. Representation of the HEC-LIFESim approach for LL estimation (modified from [44]).

The warning and evacuation module simulates the spatial redistribution of the population at risk (PAR) from its initial distribution when the warning is issued to a new distribution at the flood-arrival time. Modelling warning requires information about the hazard communication delay (i.e., time to communicate the message to the responsible authority), the warning issuance delay (i.e., time to initiate alert), the warning diffusion (i.e., time to receive the warning message), and mobilization time (i.e., time to decide on moving away from the flood area). The simulation of the evacuation process requires information about the road network, evacuation destinations, and emergency planning zones.

The loss of shelter module simulates the exposure of people in buildings during the flood event. To achieve this, first, the degree of building destruction is defined based on the local flow quantities. Based on that knowledge and local characteristics of the flood, different flood zones are assigned to buildings or levels of buildings in the locality. The three flood zones used in HEC-LIFESim were defined by McClelland and Bowles [45] as chance zone, compromised zone, and safe zone.

The loss of life module combines information about the assigned flood zone categories, the value of PAR in the corresponding zone, and the LL rate distributions to estimate life loss. The LL rate distributions were derived from historical data and reflect a physical flood environment different in each flood zone [7].

\subsection{Information Sources for the Swiss Case Study}

\subsubsection{Dam-Downstream Inhabited Locality Representative for Switzerland}

In this study, the LL model was generated for a locality downstream of a large concrete arch dam over $100 \mathrm{~m}$ in height located in Switzerland. Most Swiss dams with such characteristics are in the Cantons of Valais and Ticino. In particular, the largest valley in Canton of Valais, the Rhone Valley, could be potentially affected by a break from one of the four large arch hydropower dams (Gebidem, Mauvoisin, Moiry, and Zeuzier dams) (Figure 2). Moreover, in the upper part of the valley, there are also large gravity (the Grand Dixence dam) and embankment (the Mattmark dam) dams. Considering this potential 
for the inundation of some parts of the Rhone Valley due to a dam failure, a hypothetical locality with characteristics like those of the localities in the Rhone Valley can be considered representative for Switzerland in the LL model.

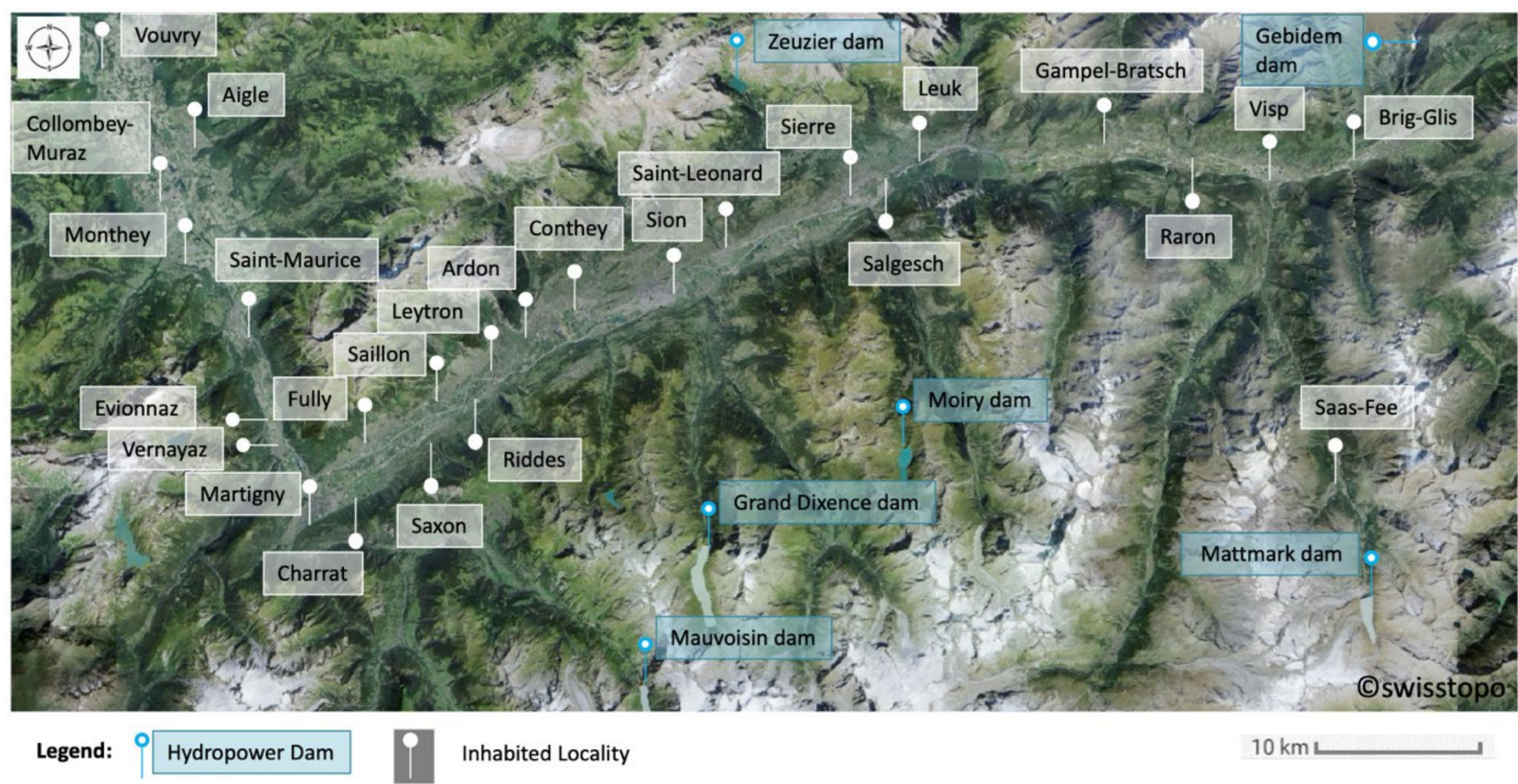

Figure 2. The Rhone Valley with all major inhabited localities, as well as large dams and dam reservoirs located around the valley (topography image is provided by swisstopo [16]).

To simulate a hypothetical locality representative for the Rhone Valley, it is important to understand which characteristics can essentially describe such a locality and, in particular, its urban part where buildings and inhabitants are concentrated. Based on a literature survey about urban analytics conducted in this study [46,47], it can be concluded that a number of characteristics related to demographics, buildings, land use, and transport are generally very important. In this study, only the characteristics significant for the dam-break LL model were considered (Table S1 in the Electronic Supplementary Material (ESI)).

The data for two demographic characteristics, namely the total population, $P_{t o t}$, and people over 65 years, $P_{065}$, were collected from the SFSO [48]. Based on the summary statistics of these data (from $1.58 \times 10^{3}$ to $2.16 \times 10^{4}$ (people) for $P_{t o t}$ and from 12.3 to 21.8 (\%) for $P_{065}$; see Table S2 in ESI), it was decided to focus only on 25 localities with a number of inhabitants $\geq 1000$ in the Rhone Valley (Table S1 in ESI).

For the chosen localities, the information related to four characteristics of the buildings and land use (Table S1 in ESI) were collected from the SFSO [49] and swisstopo [16]. Using the summary statistics of these data (Table S2 in ESI), a better understanding of the representative ranges within the selected localities in the Rhone Valley was achieved. This knowledge helped to conclude that the hypothetical locality in the current LL model should be defined using the following ranges (5-95\% quantiles):

- Ratio of building height to building area-0.017 to 0.019 (\# floors $/ \mathrm{m}^{2}$ );

- Total area of its urban part between 1.0 and $3.6\left(\mathrm{~km}^{2}\right)$;

- $\quad$ Building density-283.3 to $478.7\left(\# / \mathrm{m}^{2}\right)$;

- Fraction of residential among all buildings-92.4 to 96.3 (\%). 


\subsubsection{Swiss Representative Data for the Modules of HEC-LIFESim \\ Loss of Shelter Module}

Information needed to simulate the structural inventory for the hypothetical locality was collected from the GWR building database [49] (Figure 3). Using the description of the building category characteristics (occupancy type, structural stability criteria, and number of stories), the format of the data extracted from the GWR database was adopted to the format of the HEC-LIFESim software as shown in Table S3 in ESI. Furthermore, assumptions on the construction type of the structures were based on expert judgment (Table S3 in ESI).

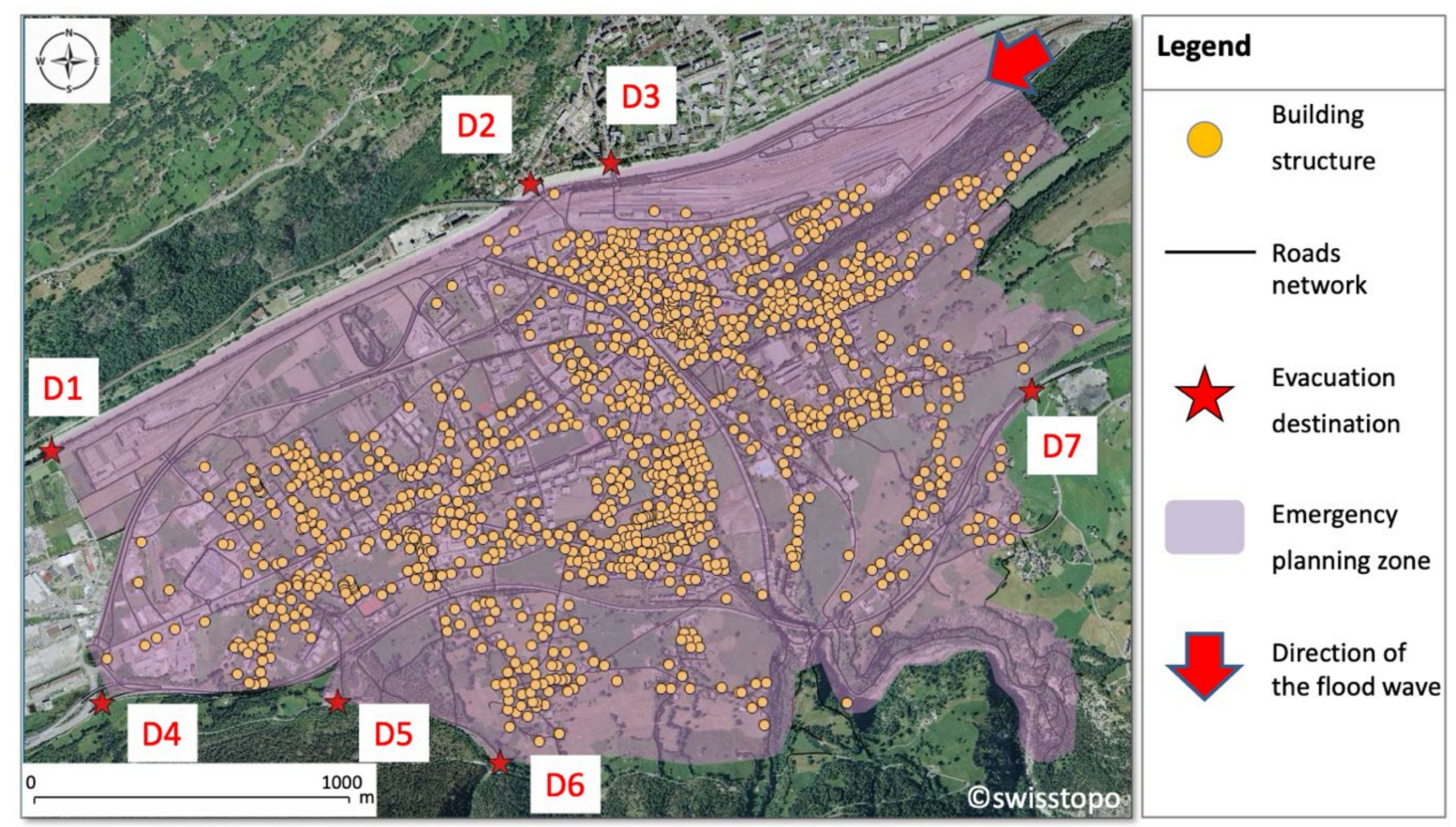

Figure 3. The building structures, road network, evacuation destinations, and emergency planning zone defined for the hypothetical locality in the LL model (topography image was provided by swisstopo [16]).

Data for the structural stability criteria of buildings in Switzerland could not be obtained for the needs of this study. Therefore, it was decided to use the data from the European Project Development of Rescue Actions [50]. In the latter, the stability criteria are given for European buildings, which can be considered as a reasonable approximation for Switzerland because of the advanced quality control and safety measures used for construction needs in the European countries [51].

Finally, time series of flow quantities at different locations of the inundated area were imported in HEC-LIFESim from the HEC-RAS [52] for three selected scenarios (see Section 3.3). In HEC-RAS and for each of the selected scenarios, the flood wave through the locality was simulated using the digital elevation data, Manning roughness coefficients, and inflow hydrograph derived from the PCE metamodel of the dam-break flood built by Kalinina, Spada, Vetsch, Marelli, Whealton, Burgherr, and Sudret [34]. For the digital elevation data of the hypothetical locality used in this study, the digital elevation model of the Brig-Glis locality was extracted from swisstopo [17] because this real locality is situated in the Rhone Valley and its buildings and land use characteristics are within the intervals given in Section 2.2.1. This choice was made to apply the proposed framework to real-world data, which better represents the Swiss conditions, rather than a simplified generic map. Furthermore, it helps to eliminate additional uncertainty due to simplification and, on the other hand, to model a more comprehensive flood dynamic. 
Warning and Evacuation Module

As introduced in Section 2.1, information about the demographics and warning-time parameters is required to simulate the warning process. For this study, the demographics data were taken from the census data provided by the SFSO [53] (Table S1 in ESI). Although the Swiss Federal Act [54] and Directive on the Safety of Water Retaining Facilities [55] provide an exhaustive description of the warning process to be used in case of the uncontrolled water release from a dam, there are no standard values for these warning-time parameters. Therefore, to understand the possible range of warning-time parameters, a literature review about dam-break LL models was conducted (see Section 4.1.2).

To simulate the evacuation, knowledge about evacuation destinations and zones is necessary. Because these data are spatial and closely linked to the elevation data, the actual evacuation destinations (D1-D7) and emergency planning zones defined for the aforementioned Brig-Glis locality by the Public Safety Department of the Municipality of Brig-Glis [56] were used for the hypothetical locality in this study (Figure 3).

Information about the road network, such as the free flow speed and number of lanes, is essential for modelling evacuation-transportation processes. In this study, road-network data were extracted from OpenStreetMap [57]. This information source was chosen since it is a free editable map of the world emphasizing local knowledge; thus, it can accurately represent the real-world local conditions.

Loss of Life Module

Finally, the intermediate results acquired from the other two modules (Figure 1) were combined with the historical LL rates to estimate life loss. The LL rates were given as curves of the frequency of exceedance with respect to the PAR. For each of the three flood zones (i.e., chance zone, compromised zone, and safe zone), an individual LL rate curve was defined.

HEC-LIFESim uses LL rates empirically derived from historical data on floods that are mostly attributable to embankment dam failures in the U.S. However, McClelland and Bowles [7] stated the importance of adjusting the historical observations underlying the LL rates to reflect study-specific characteristics of the dam type and failure mode. Therefore, alternative LL rate distributions that are representative for the topographical conditions and characteristics of dams in Switzerland developed by Kalinina et al. [58] were used in this study.

\section{Method}

In this study, metamodelling was applied for uncertainty quantification (UQ) and sensitivity analysis (SA) in the LL model for the dam-break event (Figure 4). The methodology was based on the general UQ framework introduced by Sudret [27] and De Rocquigny, Devictor, and Tarantola [28]. First, the uncertain input was defined and used as for the computational model in Section 2 (Box A in Figure 4). The computational model was then used to build the PCE-based metamodel (Box B in Figure 4). Afterwards, the uncertainty of the model output, i.e., LL estimates, was quantified by propagating uncertainty of the model input parameters through the metamodel (Box B in Figure 4). These results were compared (Box C in Figure 4) with the results obtained from the existing uncertainty model of HEC-LIFESim, given by a Monte Carlo sampling (Box A in Figure 4). Finally, using the metamodel, a global SA (GSA) could be directly performed to better understand the contributions of the uncertain model parameters to the variability of LL estimates (Box D in Figure 4). Different GSA techniques were applied in this study allowing for quality assurance of the conclusions that were drawn based on the calculated indices. 


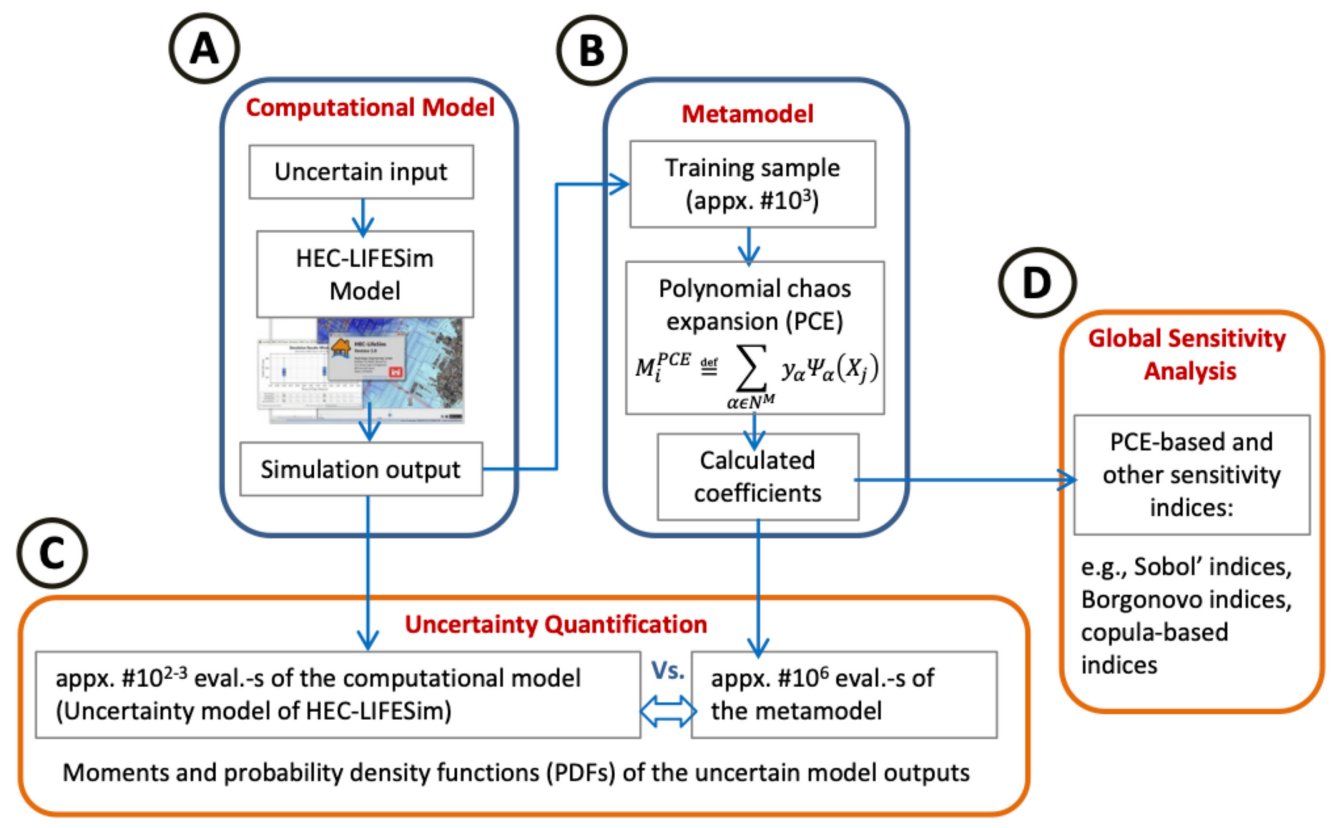

Figure 4. Methodology for uncertainty quantification and sensitivity analysis applied in this study.

\subsection{Metamodel}

\subsubsection{Uncertainty in the Model Input}

The first step to assess the uncertainty in the model output was to define the uncertainty in the model inputs (see Box A in Figure 4). In fact, input parameters for the dam-break LL model are intrinsically uncertain; therefore, for credible LL estimates, it is important to quantify and consider uncertainty in the model inputs [20]. For this purpose, the input parameters (Section 4.1) were introduced in the LL model as probability distributions. Furthermore, these distributions were defined for all possible values among the inhabited localities considered in this study (Section 2.2), thus making the metamodel generic for the selected localities rather than a model just for one locality.

Based on the availability of the collected data for the model parameters, different methods can be applied to build a probability distribution. In this study, when operating with a tiny dataset (e.g., a few data points), the maximum entropy principle was used to select the distribution family [59]. This method was chosen since it has been shown in the literature to be useful in case of scarce data to infer distributions $[34,60,61]$. In contrast, when a set of data points is available (e.g., values of the total population for the 25 considered localities), the information criteria (IC) [62,63] are calculated to test the fit of three selected probability distributions to the data. The distribution with the smallest value of IC is commonly chosen because it is a good compromise between maximizing the likelihood and minimizing model complexity. The three distributions considered in this study were uniform, beta, and lognormal. The uniform and beta distributions were considered since they are commonly used when little knowledge about the parameter is available. Furthermore, they are bounded distributions, i.e., bounds can be set based on the values among considered localities. The lognormal distribution was considered because it is commonly used for engineering applications, and comprehensive guidelines exist on how to define distribution parameters [64]. Finally, once the best fit distribution to the data among the selected three has been identified, the parameters describing the distribution, i.e., the moments, are estimated using a maximum likelihood estimator (MLE) [65].

\subsubsection{Uncertainty Propagation}

Once the uncertainty of the model inputs is defined, the uncertainty propagation step can be performed (see Box B in Figure 4). The computational LL model is defined as $=M(X)$, where $Y$ is the response of the quality of interest to the random input, $X\left(X \in \mathbb{R}^{M}\right)$, 
with a probability distribution function (PDF) $f_{X}$. In this study, this model was substituted with a computationally inexpensive analytical approximation known as metamodel. In particular, a polynomial chaos expansion (PCE) was used to build the metamodel $[29,66]$ :

$$
M^{P C E} \stackrel{\text { def }}{=} \sum_{\alpha \in N^{M}} y_{\alpha} \Psi_{\alpha}\left(X_{j}\right)
$$

where $M^{P C E}$ is the PCE metamodel response, $y_{\alpha, i}$ are real coefficients, $N$ is the sample size, $M$ is the model dimension, and the index $\alpha$ determines the degree of the multivariate polynomials $\Psi_{\alpha}$, which are the product of the underlying standard orthonormal polynomials, $\phi_{\alpha_{i}}^{(i)}$. A sample set for each model input parameter, $X_{j}$, was created by sampling the corresponding marginal distribution with the Latin hypercube sampling (LHS) technique [67].

The coefficients $y_{\alpha}$ of the PCE for a given basis can be calculated by post-processing of a training sample of the input random vector, $\mathcal{X}=\left\{x^{(1)}, \ldots, x^{(N)}\right\}, x^{(i)}=\mathbb{R}^{M}$, the experimental design (ED), and the corresponding set of model responses, $\mathcal{Y}=\left\{\boldsymbol{y}^{(1)}, \ldots, \boldsymbol{y}^{(N)}\right\}$ :

$$
y_{\alpha}=\operatorname{argmin} \frac{1}{N} \sum_{i=1}^{N}\left[\boldsymbol{y}^{(i)}-\sum_{\alpha \in N^{M}} y_{\alpha} \Psi_{\alpha}\left(x^{(i)}\right)\right]^{2}
$$

When calculating the coefficients for high-degree expansions and a limited ED, such as in the applied model, overfitting can occur. To avoid potential overfitting, least angle regression methods (LARS, [68]) for the calculation of the coefficients can be used. When the PCE coefficients are calculated, the PCE model can be used as a predictor of new points outside of the ED.

In this study, the construction of the metamodel was performed using PCE based on LARS in UQLab, an uncertainty quantification toolbox for MATLAB $[69,70]$.

More detailed information on the theoretical background of PCE-based metamodels was provided by Blatman and Sudret [71]. Furthermore, a similar application of the metamodelling approach for uncertainty quantification in the dam-break-related model was presented by Kalinina, Spada, Vetsch, Marelli, Whealton, Burgherr, and Sudret [34] and used as input for the definition of the flood scenarios (see Section 3.3) in this study.

\subsubsection{Validation of the Metamodel}

The agreement between the original model and the constructed PCE model was assessed using the normalized leave-one-out cross-validation error $\left(\epsilon_{L O O}\right)$ [72]:

$$
\epsilon_{L O O} \stackrel{\text { def }}{=} \frac{\sum_{i=1}^{K}\left(M\left(x^{(i)}\right)-M^{P C E \backslash i}\left(x^{(i)}\right)\right)^{2}}{\left.\sum_{i=1}^{K}\left(M\left(x^{(i)}\right)-\hat{\mu}_{Y}\right)\right)^{2}}
$$

where $M\left(x^{(i)}\right)$ is the evaluation of the computational model, $\hat{\mu}_{Y}$ is the mean value of the computational model, and $M^{P C E \backslash i}\left(x^{(i)}\right)$ is the PCE.

In total, $\mathrm{K}$ different metamodels were built with each metamodel being constructed on a reduced $\operatorname{ED}\left\{x^{(i)}=\left\{x^{(j)}, j=1, \ldots, K, j \neq i\right\}\right.$, except the ith observation. Afterwards, this observation was used as a single validation point for this metamodel.

Finally, the mean square error (MSE) was estimated and normalized by the estimated variance of the computational model to evaluate the convergence of the mean values of the model outputs. For that, ED was divided into two samples: one sample was the same sample set used as validation sample, $X^{V A L}$, and the other was used for the construction of the PCE, $X^{N E D}$. Then, the validation error was estimated using the metamodel prediction, $Y^{N E D}$, and the computational model output, $Y^{V A L}$ : 


$$
M S E=\frac{\sum_{i=1}^{n}\left(Y_{i}^{N E D}-Y_{i}^{V A L}\right)^{2}}{\sum_{i=1}^{n}\left(Y_{i}^{V A L}-\bar{Y}^{V A L}\right)^{2}}
$$

\subsection{Global Sensitivity Analysis}

To meet the objectives of this study, the sensitivity of the model output, $Y$, to each model input parameter was quantified (see Box D in Figure 4). This was done with the use of the sensitivity indices indicating the contribution of each model input to the overall uncertainty of the output. To perform the sensitivity analysis, the preference was given to a GSA rather than to LSA because global methods consider the entire input domain. Two GSA methods, namely, 1st order and total Sobol' indices and Borgonovo indices, were considered in this study allowing for quality assurance of the conclusions made from the sensitivity analysis. Although other GSA methods could be applied, the choice of these two methods was driven by the fact that they benefit from the constructed metamodel (Section 3.1) and calculate the sensitivity coefficients using previously estimated PCE coefficient without additional sampling of the original computational model [73]. The remainder of this section provides a concise theoretical background for the two GSA methods.

\subsubsection{PCE-Based Sobol' Indices}

The Sobol' method is a variance-based sensitivity analysis that aims at understanding how the output variance can be attributed to individual model inputs and the interaction between inputs [74,75]. Thus, it decomposes the total variance $D$ of the model response, $Y$, (also defined as a function of $X, Y=M(X)$ ) into contributions from each input parameter $\left(X_{j}, j=1 \ldots n\right)$ and from interactions between parameters. The Sobol' decomposition can be expressed as:

$$
M(x)=M_{0}+\sum_{i=1}^{s} M_{i}\left(X_{i}\right)+\sum_{i=1}^{s} \sum_{i \neq j}^{s} M_{i j}\left(X_{i}, X_{j}\right)+M_{1 . . s}\left(X_{1}, X_{2}, \ldots X_{s}\right)
$$

where $M_{0}$ and $D$ are the mean and variance of a random variable, $M(X)$, and the $D$ can be decomposed further as:

$$
D=\sum_{i=1}^{M} D_{i}+\sum_{1 \leq i<j \leq M} D_{i j}+\ldots+D_{12 \ldots M}
$$

The Sobol' sensitivity indices are then defined as the ratio of the partial variance, $D_{i}$, to the total variance, $D[76]$ :

$$
S_{i_{1} \ldots i_{s}}=\frac{D_{i_{1} \ldots i_{s}}}{D}
$$

In particular, the first-order Sobol' index, $S_{i}$, represents the fraction of the total variance, $D$, attributable to an effect of a single input variable, $D_{i}$, i.e., $S_{i}=D_{i} / D$. The total Sobol' index, $S_{T_{i}}$, is the sum of all sensitivity indices (i.e., $S_{T_{i}}=S_{i}+S_{i j}+\ldots+S_{1 . . i . . s}$ ), accounting for both the first- and higher-order effects.

Commonly, Sobol' indices are calculated using a Monte Carlo approach, which, in case of high dimensional models, is computationally expensive. In contrast, PCE-based Sobol' indices take advantage of a surrogate-model technique to extract the most accurate information based on a relatively small sample size [77]. The calculation principle is based on the similarity between the Sobol' decomposition (Equation (5)) and the PC expansion (Equation (8)):

$$
M(x)=\sum_{\alpha \in N^{M}} y_{\alpha} \Psi_{\alpha}(x)=M_{0}+\sum_{\left\{i_{1}, \ldots, i_{s}\right\} \subset\{1, \ldots, M\}} \sum_{\alpha \in A_{\left\{i_{1} \ldots i_{s}\right\}}} y_{\alpha} \Psi_{\alpha}(x)
$$


By unicity of the Sobol' decomposition, the definition $M_{i_{1} \ldots i_{s}}\left(x_{i_{1}}, \ldots, x_{i_{s}}\right) \stackrel{\text { def }}{=} \sum_{\alpha \in A_{i}} \mathrm{y}_{\alpha} \Psi_{\alpha}(\boldsymbol{x})$ applies. Furthermore, the partial variances $\left.D_{i_{1} \ldots i_{s}}=\operatorname{Var}\left[M_{i_{1} \ldots i_{s}}(X)\right)\right]$ can be calculated by summing up the squared PC coefficients, i.e., the first-order term as $D_{i}=\sum_{\alpha \in A_{i}} y_{\alpha}^{2}$, where $A_{i}=\left\{\alpha \in N^{M}: \alpha_{i}>0, \alpha_{j \neq i}=0\right\}$ with $\mathrm{N}$ being the number of samples in the ED and M being the dimension of the model, and high-order terms as $D_{i_{1} \ldots i_{s}}=\sum_{\alpha \in A_{\left\{i_{1} \ldots i_{s}\right\}}} y_{\alpha}^{2}$, where $A_{i_{1} \ldots i_{s}}=\left\{\alpha \in N^{M}: k \in\left\{i_{1} \ldots i_{s}\right\} \Leftrightarrow \alpha_{i}>0\right\}$.

Therefore, the first-order Sobol' indices can be computed as:

$$
S_{i}=\sum_{\alpha \in A_{i}} y_{\alpha}^{2} / D, A_{i}=\left\{\alpha \in N^{M}: \alpha_{i}>0, \alpha_{j \neq i}=0\right\}
$$

and the total Sobol' indices are:

$$
S_{T_{i}}=\sum_{\alpha \in A_{T_{i}}} y_{\alpha}^{2} / D, A_{T_{i}}=\left\{\alpha \in N^{M}: \alpha_{i}>0\right\}
$$

\subsubsection{Borgonovo Indices}

Borgonovo indices, $\delta_{i}$ [40], consider the distance between the output distribution, $f_{Y}$, and the output distribution conditional to one or several inputs, $f_{Y \mid X_{i}}$. If the value of $\delta_{i}$ is close to zero, then the variable is unimportant; on the other hand, the larger the value of $\delta_{i}$ is, the more important the variable is.

In other words, $\delta_{i}$ index of a random input variable $X_{i}$ is a measure of the expected shift in the probability distribution of the model output, when $X_{i}$ is set to a fixed value:

$$
\delta_{i}=\frac{1}{2} \int f_{X_{i}}\left(x_{i}\right) \int\left|f_{Y}(y)-f_{Y \mid X_{i}}\left(y \mid x_{i}\right)\right| d y d x_{i}
$$

In Equation (11), $f_{Y \mid X_{i}}$ is the conditional probability distribution function (PDF) that can be approximated by drawing a sample of the input vector $X$ of size $\mathrm{K}$ and afterwards binning the variable $X_{i}$ in classes. For this, the $P$ disjoint ranges $\left\{a_{p}, b_{p}\right), a_{p}>b_{p}$ on the $X_{i}$ variable are defined. The input sample is then divided in subsets $C_{p}=$ $\left\{x^{(j)}\right.$, such that $\left.a_{p} \leq x_{i}^{(j)} \leq b_{p}\right)$ and the $f_{Y}$ investigated in each class such as $f_{Y \mid X_{i}=x_{i}}(y) \approx$ $\hat{f}_{Y \mid C_{p} \supset x_{i}}(y)$.

In this study, the Borgonovo indices, $\delta_{i}$, are assessed by using the histogram-based estimation principle [73]. First, a sample of the input vector $X$ of size $K, \mathcal{X}=\left\{x^{(1)}, \ldots, x^{(K)}\right\}$ is generated, and afterwards, the corresponding set of model responses, $\mathcal{Y}=M\left(x^{(1)}\right), \ldots$, $M\left(x^{(K)}\right)$, is evaluated. The unconditional PDF is approximated directly from the histogram of $\mathcal{Y}$, while to define the conditional PDF, a histogram is set in each class. It is recommended to have enough samples in each class (e.g., $K \geq 10^{3}$ ) to be able to accurately estimate the conditional PDFs with histograms [73].

By computing the sum of absolute differences between the unconditional and conditional PDFs, the inner integral in Equation (11) is calculated. Afterwards, the sum of the absolute difference between the conditional and unconditional PDF values is calculated for each class and subsequently averaged by taking the mean of the estimates. This yields an estimation of the outer integral in Equation (11).

\subsection{Definition of the Scenarios}

The uncertainty in the model input parameters, which are likely affecting the LL estimates, was quantified in this study. In addition, different scenarios were modelled to understand the influence of different time and flood conditions on the LL estimates. In particular, six scenarios were defined as combinations of two different times of the 
day (daytime (2 p.m.), night-time (2 a.m.)) and three different flood severities (F05, F50, F95). The time of the day reflects different distributions of people among buildings and the ability of people to react to changing conditions (Table S3 in ESI). Furthermore, as described in Section 2.2, flow characteristics (e.g., water depth, velocity) for different floodseverity scenarios were modelled in HEC-RAS using the dam-break outflow hydrographs computed with the PCE metamodel built by Kalinina, Spada, Vetsch, Marelli, Whealton, Burgherr, and Sudret [35]. Three different outflow hydrographs were built using the median (scenario F50), 5\% (scenario F05), and 95\% quantile (scenario F95) values of the generic PCE metamodel of the dam-break outflow hydrograph reflecting Swiss conditions (Table 1). Maps of the resulting maximal flow depth and velocity are provided in Table S4 in ESI.

Table 1. Three different dam-break outflow hydrographs $\left(\mathrm{m}^{3} / \mathrm{s}\right)$ representing three different scenarios of the flood severity.

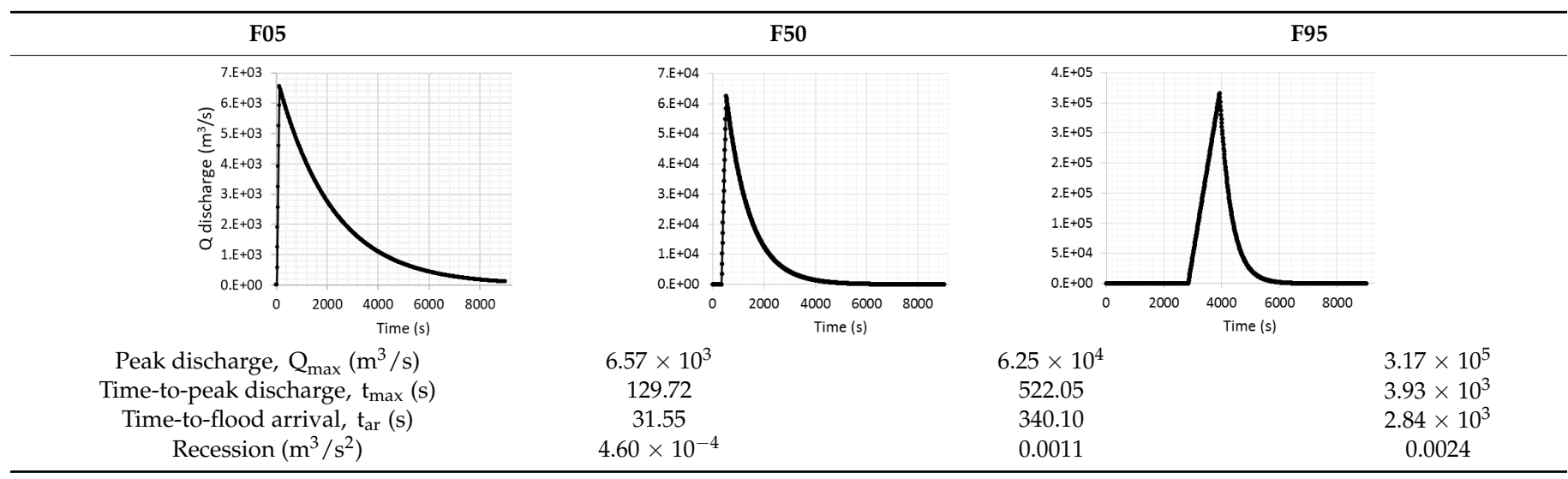

\section{Results and Discussion}

\subsection{Uncertainty in Model Inputs}

\subsubsection{Definition of the Sources of Uncertainties}

Sources of uncertainty identified in the LL model can be allocated to two categories, namely aleatory (due to natural variability, e.g., failure mode, time of the day, warning parameters) and epistemic uncertainty (due to lack of knowledge, e.g., flow quantities in the inundation area, share of people who decided to evacuate) [78]. Based on expert opinion and studies conducted in the past (Table S5 in ESI), several model input parameters were selected in this study. This selection aimed to include those parameters that can be a significant source of variability for the LL model output and that are generally part of dam risk management and, thus, can be adjusted by dam-safety specialists.

As a result, three characteristics of the receptor (i.e., the inhabited locality) and four characteristics of its reaction on the flood load (defined in Section 3.3) were chosen and their uncertainties were explicitly modelled (Table 2).

Table 2. Input parameters for the LL model.

\begin{tabular}{|c|c|c|c|}
\hline Parameter & Name & Unit & Definition \\
\hline & & Receptor & \\
\hline$P_{\text {tot }}$ & Total population & \# people & Total population of the modelled inhabited locality \\
\hline$P_{065}$ & Population over 65 & fraction & Part of the total population older than 65 years \\
\hline \multirow[t]{2}{*}{$H$} & Building foundation height & $\mathrm{m}$ & $\begin{array}{c}\text { Height between the level of the ground and the level of the ground } \\
\text { floor in the building }\end{array}$ \\
\hline & & Reaction & \\
\hline$F_{\text {chance }}$ & Fatality rate in the chance zone & fraction & $\begin{array}{c}\text { Fatality rate in the chance zone given as a part of PAR that will lose } \\
\text { their life }\end{array}$ \\
\hline$F_{\text {compr }}$ & Fatality rate in the compromised zone & fraction & $\begin{array}{l}\text { Fatality rate in the compromised zone given as a part of PAR that } \\
\text { will lose their life }\end{array}$ \\
\hline$T_{h c d}$ & Hazard communication delay & $\mathrm{h}$ & $\begin{array}{l}\text { Time that it takes the dam operator to communicate the message to } \\
\text { the local authorities }\end{array}$ \\
\hline$T_{\text {wid }}$ & Warning issuance delay & $\mathrm{h}$ & Time that it takes the local authorities to initiate warning \\
\hline
\end{tabular}




\subsubsection{Quantification of the Uncertainties in the Input Parameters}

To build marginal distributions for the parameters of the hypothetical locality (receptor), namely $P_{\text {tot }}$ and $P_{065}$ (Table 2), the data of 25 major localities in the Rhone Valley (Table $\mathrm{S} 1$ in ESI) were extracted from the SFSO [48]. The AIC coefficients were calculated for three types of probability distributions to assess the best fit to the extracted data (Section 3.1). For $P_{t o t}$, the smallest AIC was estimated for a lognormal distribution and for $P_{065}$ for a beta distribution (Figure 5). For the lognormal distribution defined for $P_{t o t}$, truncation was considered to prevent obtaining unrealistically large values when sampling the tail of the modelled distribution. For the building foundation height, $H$, no information was available about more or less likely values to quantitatively describe the uncertainty of this parameter. Therefore, it was assumed that $H$ follows a uniform distribution defined in the range between $0.2(\mathrm{~m})(0.65 \mathrm{ft})$ and $1.5(\mathrm{~m})(5 \mathrm{ft})$.
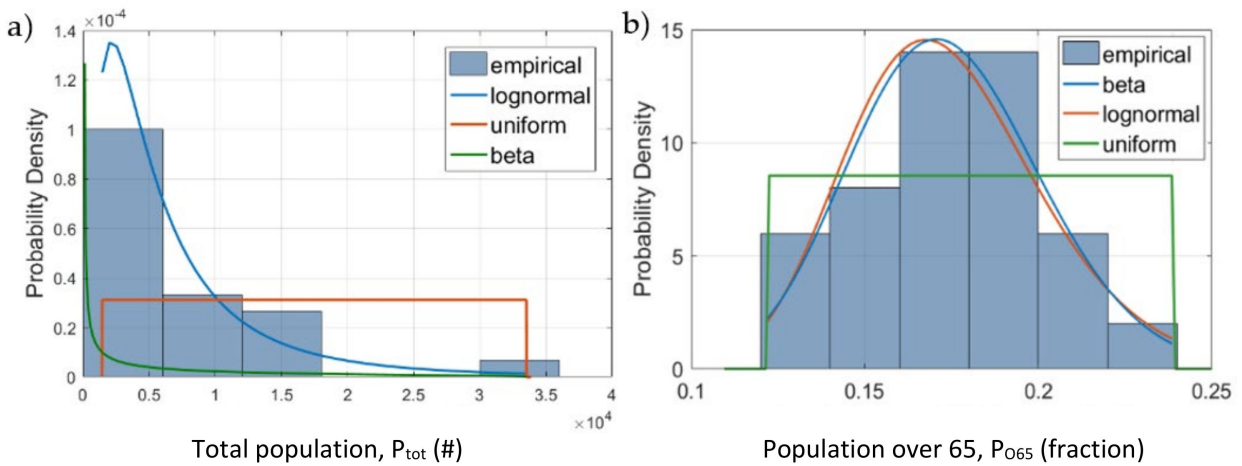

Figure 5. Marginal distributions for the (a) total population, $P_{t o t} ;(\mathbf{b})$ population over 65 years, $P_{065}$.

As described in Section 2.1, HEC-LIFESim uses three flood zones, namely, chance, compromised, and safe zone. In the current LL model, the safe zone was considered with a zero probability for fatality (i.e., zero fatality rate). The values for the fatality rates for both the chance, $F_{\text {chance }}$, and compromised, $F_{\text {compr }}$, zones were extracted from the data for concrete dams in the OECD countries collected by Kalinina, Spada, and Burgherr [58]. Based on the available values and using the maximum entropy principle (Section 3.1), the distributions for $F_{\text {chance }}$ and $F_{\text {compr }}$ were defined as uniform ones as shown in Figure 6.
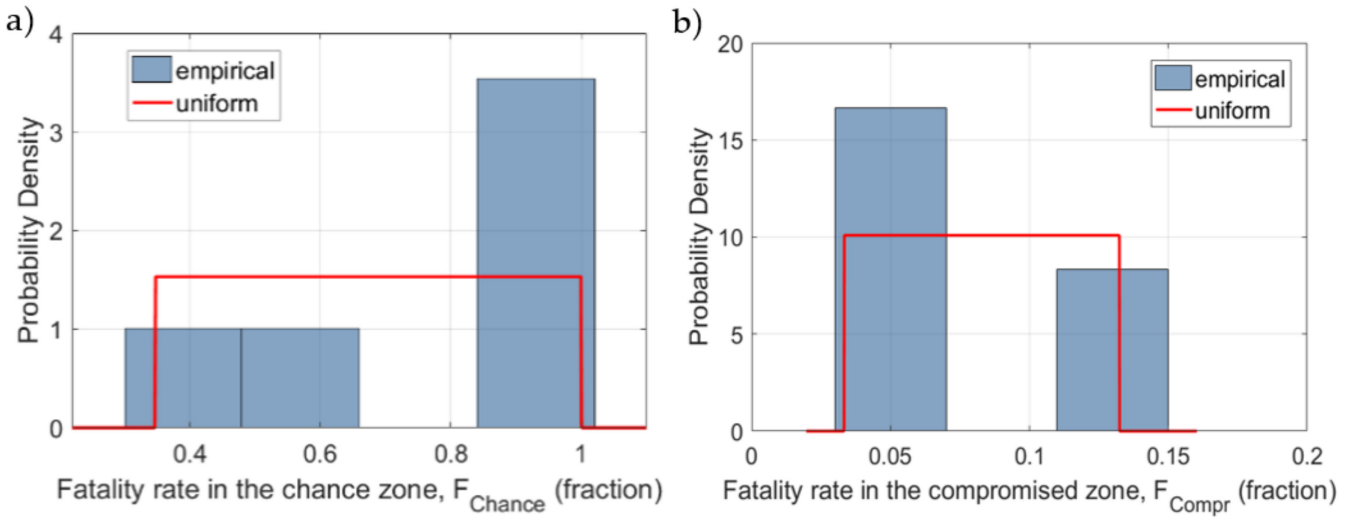

Figure 6. Marginal distributions for (a) fatality rate in the chance zone, $F_{\text {chance }}$; (b) fatality rate in the compromised zone, $F_{\text {compr }}$.

An overview of previous studies on dam-break LL models was conducted to determine possible values for the parameters $T_{h c d}$ and $T_{\text {wid }}$. The reviewed studies addressed warning time (defined as a time between alarm initiation and imminent hazard) but not $T_{\text {wid }}$. The warning time is generally defined between $2 \mathrm{~h}$ before and $2 \mathrm{~h}$ after the dam break (Table 3). 
Table 3. Values for warning time used in various studies conducted on the topic of the dam-break modelling.

\begin{tabular}{ccc}
\hline Author (Year) & Paper Title & Warning Time (h) \\
\hline DeKay and McClelland [5] & $\begin{array}{c}\text { Predicting loss of life in cases of dam } \\
\text { failure and flash flood }\end{array}$ & from -4 * to 0 \\
\hline Graham [79] & $\begin{array}{c}\text { A Procedure for Estimating Loss of Life } \\
\text { Caused by Dam Failure }\end{array}$ & from -1 to 0 \\
\hline Darbre [2] & Dam Risk Analysis & from 0.25 to 0.5 \\
\hline Bowles and Aboelata [12] & $\begin{array}{c}\text { Evacuation and life-loss estimation } \\
\text { model for natural and dam break floods } \\
\text { Wang et al. [80] }\end{array}$ & $\begin{array}{c}\text { Life Loss Estimation Based on } \\
\text { Dam-Break Flood Uncertainties and } \\
\text { Lack of Information in Mountainous } \\
\text { Regions of Western China }\end{array}$ \\
\hline
\end{tabular}

To reflect this in our LL model, it was assumed that the dam operator would know about the hazard $2 \mathrm{~h}$ before the dam break (constant across all considered scenarios), but the authorities would react with different $T_{\text {wid }}$. In this study, $T_{\text {wid }}$ was described by a uniform distribution in the range of $0-4 \mathrm{~h}$ starting from the hazard identification (blue solid line in Figure 7), thus making the alarm initiation be in the range between $2 \mathrm{~h}$ before and $2 \mathrm{~h}$ after the dam break (Figure 7).

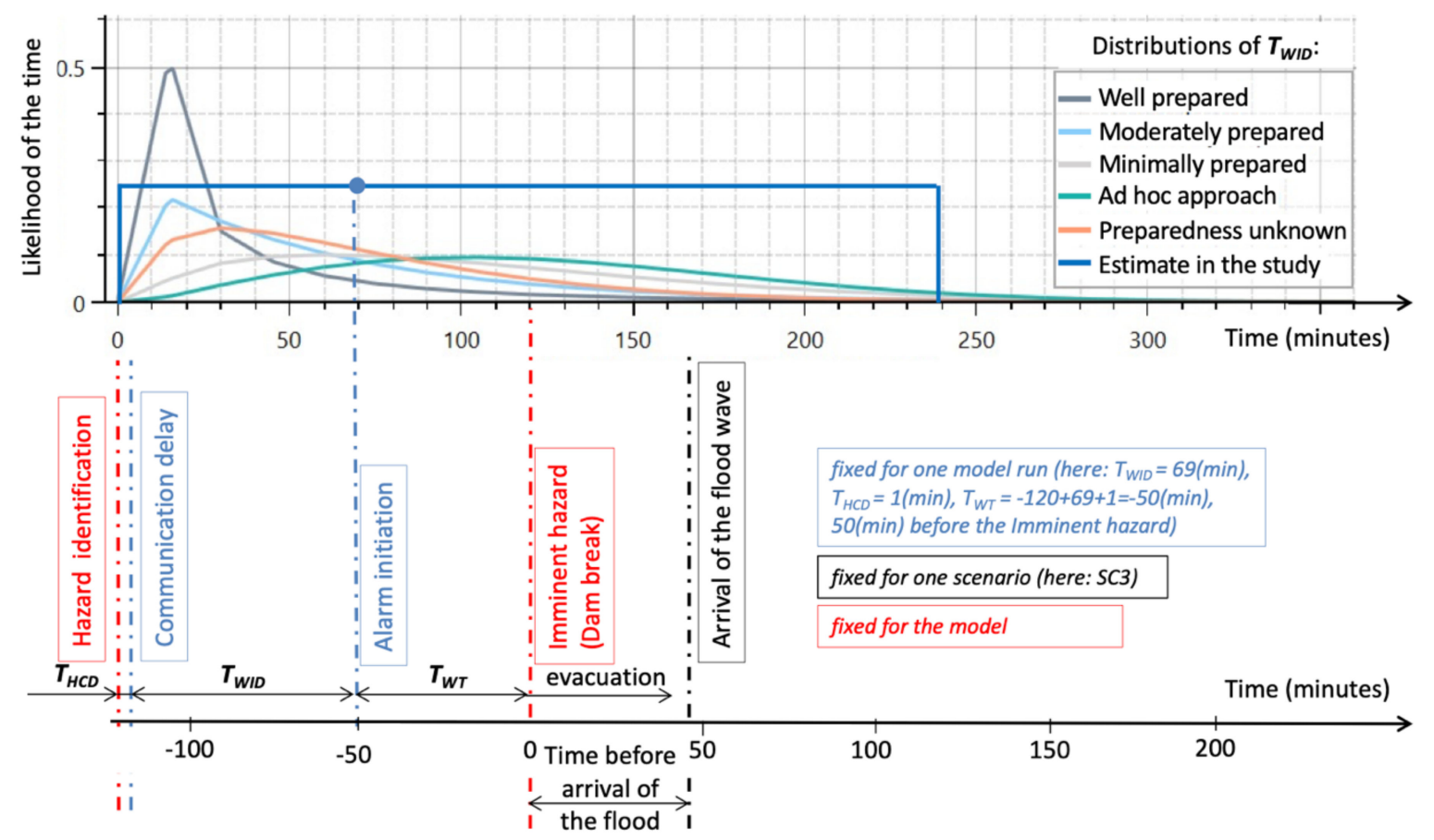

Hazard communication delay $\left(T_{H C D}\right)$ - Time that it takes the dam operator to communicate the message to the local authorities Warning issuance delay $\left(T_{\text {WID }}\right)$ - Time between Hazard identification and Alarm initiation Warning time $\left(\boldsymbol{T}_{\boldsymbol{W}}\right)$ - Time between Alarm initiation and Imminent hazard

Figure 7. Time diagram for warning and evacuation in the dam-break event and with distributions for $T_{\text {wid }}$ (default in HEC-LIFESim or specific for this study); all time parameters are given in (min) to help visualization.

In addition to $T_{w i d}$, the time of the alarm was defined by the communication delay between the dam operator and authorities, $T_{h c d}$. In the reviewed studies, no information 
was provided for $T_{h c d}$. Therefore, $T_{h c d}$ was assumed to be uniformly distributed between 0 (h) and 0.25 (h) based on expert judgment. For both $T_{h c d}$ and $T_{\text {wid }}$, a uniform distribution was assumed based on a conservative assumption because there was little confidence in the mean and median of data in the defined range. Finally, due to the different nature of the uncertain model inputs and the fact that some of them were defined based on assumptions, no correlation was modelled between the input parameters.

For all seven marginal distributions assessed in this study, the calculated parameters, moments, and truncation ranges are given in Table 4.

Table 4. Information about the marginal distributions specified for the input of the metamodel.

\begin{tabular}{cccccc}
\hline Parameter & Unit & Distribution & Hyper-Parameters & Truncation & Mean and Variance \\
\hline & & & Receptor & \\
$P_{\text {tot }}$ & \# people & $P_{\text {tot }} \sim \operatorname{Lognorm}(\mu, \sigma)$ & $\mu=8.50, \sigma=0.90$ & {$[140034,000]$} & $7.35 \times 10^{3}, 8.21 \times 10^{3}$ \\
$P_{\text {o65 }}$ & fraction & $P_{o 65} \sim \operatorname{Beta}(\alpha, \beta)$ & $\alpha=33.08, \beta=156.90$ & - & $0.17,0.027$ \\
$H$ & $\mathrm{~m}$ & $H \sim \mathrm{U}(\mathrm{a}, \mathrm{b})$ & $\mathrm{a}=0.2, \mathrm{~b}=1.5$ & - & $0.85,0.38$ \\
& & & $\operatorname{Reaction}$ & - & $0.67,0.19$ \\
$F_{\text {chance }}$ & fraction & $F_{\text {chance }} \sim \mathrm{U}(\mathrm{a}, \mathrm{b})$ & $\mathrm{a}=0.35, \mathrm{~b}=1$ & $0.083,0.029$ \\
$F_{\text {compr }}$ & fraction & $F_{\text {compr }} \sim \mathrm{U}(\mathrm{a}, \mathrm{b})$ & $\mathrm{a}=0.033, \mathrm{~b}=0.13$ & - & $2,1.15$ \\
$T_{\text {wid }}$ & $\mathrm{h}$ & $T_{\text {wid }} \sim \mathrm{U}(\mathrm{a}, \mathrm{b})$ & $\mathrm{a}=0, \mathrm{~b}=4$ & - & $0.13,0.072$ \\
$T_{\text {hcd }}$ & $\mathrm{h}$ & $T_{\text {hcd }} \sim \mathrm{U}(\mathrm{a}, \mathrm{b})$ & $\mathrm{a}=0, \mathrm{~b}=0.25$ & - & - \\
\hline
\end{tabular}

\subsection{Uncertainty in Model Output}

For each of the six scenarios (Section 3.3), a generic PCE metamodel was calculated using the ED obtained from 550 runs of the original computational model. This value was selected based on the calculation of both $\epsilon_{L O O}$ and MSE (see Section 3.1) for different sizes of the ED as shown in Figure 8 and Table S6 in ESI. To calculate MSE, the experimental design (ED) was divided into a validation sample, $X^{V A L}$, i.e., the same sample set of 100 points, and the sample used for construction of the PCE, $X^{N E D}$, i.e., from 50 to 550 sample points.
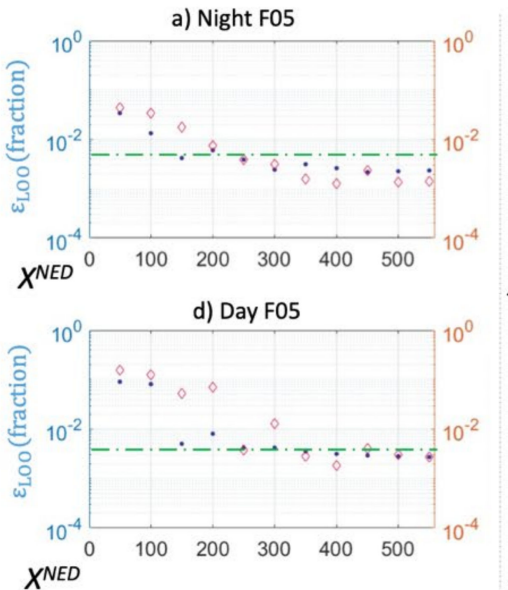

-. - - Value of the 5 -fatalities threshold
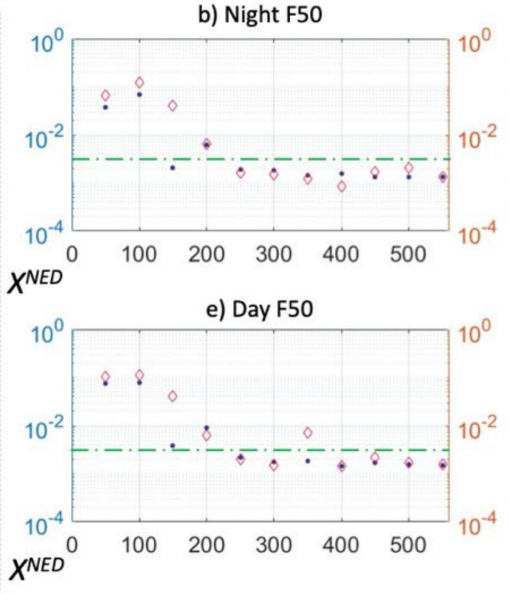

- Leave-one-out error, $\epsilon_{L O o}$
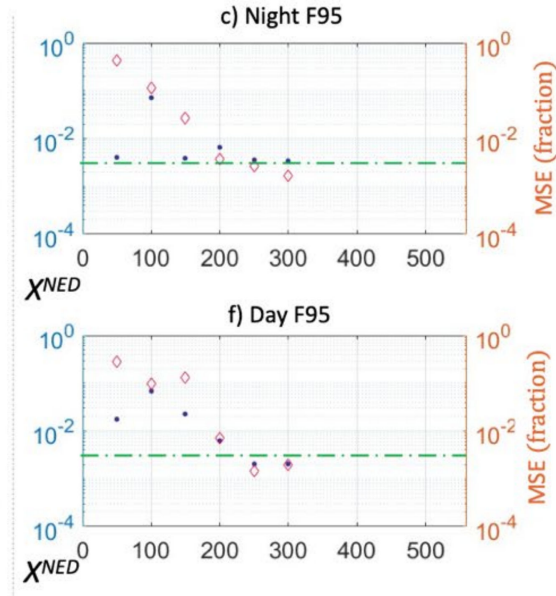

$\diamond$ Mean square error, MSE

Figure 8. The value of $\epsilon_{L O O}(\%)$ and MSE error (\%) calculated for the LL estimates obtained in each of the six defined scenarios using different sizes of the experimental design.

The PCE degree shown in Table S6 in ESI is the degree of the PCE with the lowest $\epsilon_{L O O}$ in the array of the given degrees (i.e., 1-15) [71]. The results for both $\epsilon_{L O O}$ and MSE showed that, with the increasing sample size of ED, the value of the errors decreases.

In general, for the increase of the ED from 50 to 250 samples, the decrease of the MSE and $\epsilon_{L O O}$ value is well-pronounced. With the further growth of the $X^{N E D}$ sample size, the values of both errors remain rather stable; however, there is still a tendency of $\epsilon_{L O O}$ and MSE to decrease. Therefore, to keep the balance between the metamodel accuracy and 
computational costs in this study, the maximal size of the ED was limited to 550 samples. This choice was based on the fact that, at this size of the ED, errors had become smaller than an error of five fatalities, which corresponds to a severe accident according to the definition given by Hirschberg et al. [81]. In contrast, in the night-time and daytime F95 scenarios, a smaller $E D$ (300 samples) is required to reach the threshold of an error of five fatalities. With the largest water depths reached in these two scenarios, it may be that even by changing values of the other input parameters (e.g., $P_{065}$ or $H$ ), the model output remains at the maximal LL value. Hence, no large variation is shown in the LL estimates, and the metamodel converges faster.

The constructed PCE from the ED was then evaluated on the new sample set of size $1,000,000$ using the coefficients calculated in the previous step. In Figure 9, the histogram of the computational model response, $Y^{E D}$, and the histogram of the PCE metamodel response, $M^{P C E}$, are shown for each scenario.

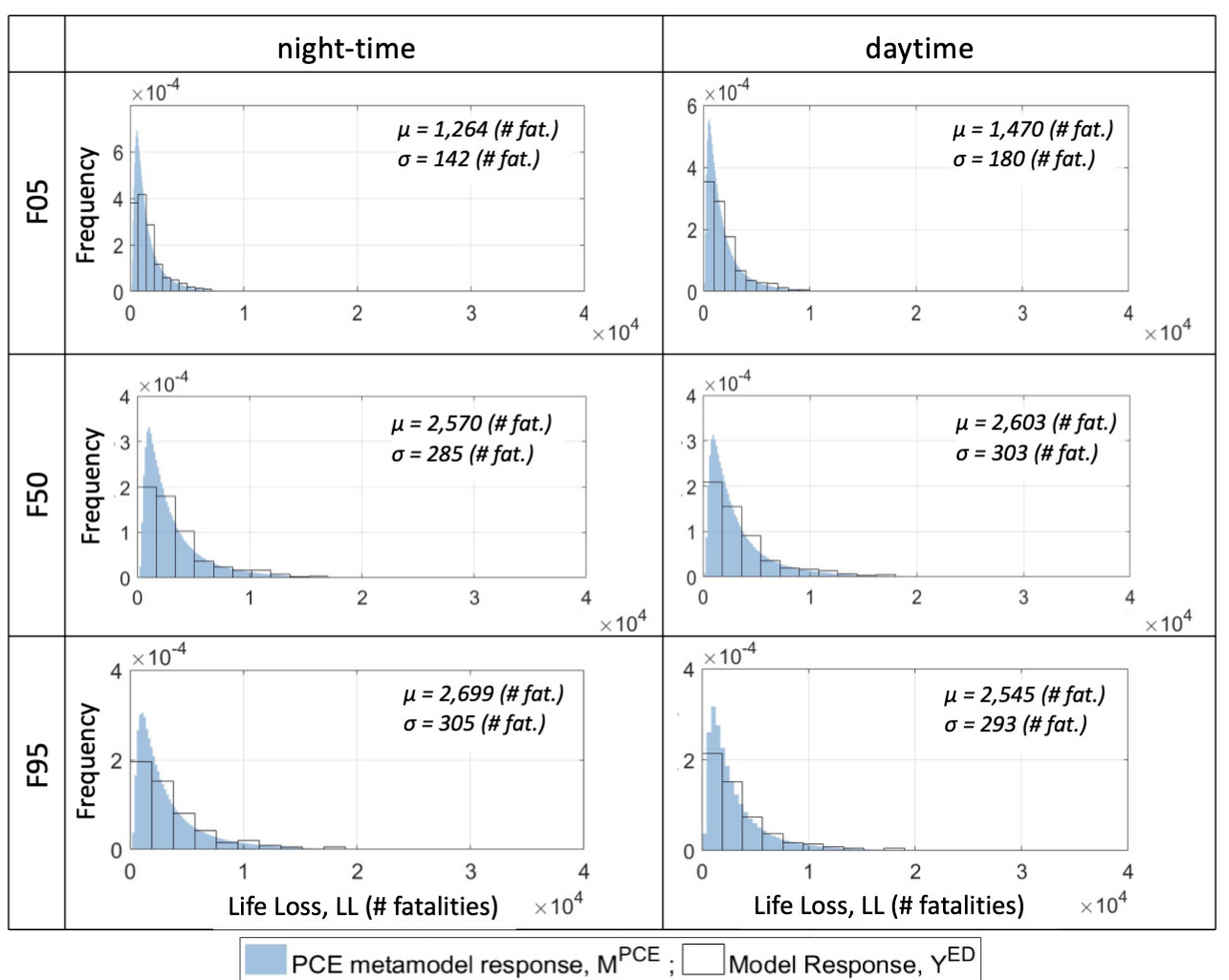

Figure 9. Model response to the experimental design, $\mathrm{Y}^{\mathrm{ED}}$, and PCE response, $\mathrm{M}^{\mathrm{PCE}}$, of the LL estimates obtained in each of the six defined scenarios.

The histograms together with the calculated moments of the metamodel response reflect the variability of the LL estimates between different scenarios. When comparing $M^{P C E}$ among scenarios, the mean value for LL estimates of F05 and F50 inflow hydrographs is higher for the daytime than for the night-time scenario (1470 (F05) and 2603 (F50) fatalities versus 1264 (SF05) and 2570 (F50) fatalities). Although, in general, historical dam breaks at night-time resulted in higher LL, in the current LL model, a substantial part of the inundated area in F50 and F05 is covered with commercial buildings, which are at daytime heavily populated with inhabitants. At night-time, all these buildings are empty, and all people are in their residential buildings placed in the elevated locations. In contrast, in F95, estimates for the night-time are higher than the daytime (2699 versus 2545 fatalities). This can be explained by the fact that, in more severe inundation scenarios (F95), the slower night-time response of the PAR to warnings becomes relevant.

When comparing the scenarios with the various flood severities (F05, F50, F95), differences in the model results can be observed. Therefore, flow quantities of the dam-break 
flood (i.e., water depth, flow velocity) are influential for the LL estimates. This is also supported by the fact that the Swiss guidelines, which are employed currently for assessing the consequences of a dam break [82], use the water depth and velocity (along with the flood arrival time) as the input for their calculations.

Among the six scenarios, the lowest LL estimates are estimated for F05 during both times of the day because a smaller area of the locality is inundated compared to the other two inflow hydrographs. Additionally, water depth and flow velocities are the smallest for these two scenarios (Table S4 in ESI). The difference between results for F50 and F95 is almost negligible. Although the maximal water depths calculated in F95 are the largest among scenarios, two factors likely counteract a substantially larger LL in F95 than in F50. First, the maximal flow velocity calculated in F95 $(15 \mathrm{~m} / \mathrm{s})$ is in the same order of magnitude as the one in F50 $(21 \mathrm{~m} / \mathrm{s})$. Second, the inflow hydrograph for F95 calculated from the PCE metamodel has a much larger value for $t_{a r}$ (Table 1), which means that the flood wave in F95 arrives at the locality much later than in F50, giving people more time for evacuation.

\subsection{PCE and Monte Carlo LL Estimates Comparison}

The LL estimates, including their uncertainty, resulting from the proposed method and the Monte Carlo (MC) sampling from HEC-LIFESim are compared in Table S7 in ESI.

In all considered scenarios (F05, F50, and F95) and for both considered times ( 2 a.m. and 2 p.m.), a good agreement is found for the LL estimates between the PCE metamodel and the MC sampling. However, this statement is only valid for large sample sizes of the MC model, i.e., when the model started to converge (between 10,000 and 100,000 sampling). This comparison validated the results of the metamodel since they are in line with the MC results from HEC-LIFESim.

Finally, the main difference between the PCE and the MC models is related to the computational costs of the two methods. In fact, on the same machine, the MC model required $\sim 2$ (for 10,000 samples) to $\sim 20 \mathrm{~h}$ to run, i.e., to obtain stable results, while for the metamodel, the experimental design (ED) required $\sim 20 \mathrm{~min}$ and the run of the PCE for $1,000,000$ times only a few seconds. Therefore, this clearly shows the improvement in terms of computational costs of the PCE method proposed in this study with respect to the MC model implemented in HEC-LIFESim.

\subsection{Global Sensitivity Analysis: Impact of Model Inputs on LL Estimates}

The sensitivity-analysis methods chosen in this study can benefit from the PCE approach and reduce the cost of GSA by calculating sensitivity indices from the PCE coefficients without the need for additional sampling.

The Sobol' indices were calculated for all model input parameters in the night-time and daytime F50 scenarios (Figure 10). For the night-time case, the first-order Sobol' indices, $S_{i}$, indicate that, among the inputs, the population in the locality, $P_{t o t}$, fatality rate in the chance zone, $F_{\text {chance }}$, and warning issuance delay, $T_{\text {wid }}$, contributed most to the variability of the LL estimates. This result is in line with the conclusions given in the overview report "Dam Risk Analysis" [2] produced by the Swiss Federal Office for Water and Geology (currently Swiss Federal Office for the Environment) where the population at risk (which is directly related to $P_{t o t}$ ) and the timeliness of dam failure warning (and $T_{\text {wid }}$ being part of this timeliness) are among the factors influencing LL.

On the other hand, fatality rate in the compromised zone, $F_{c o m p r}$, communication delay, $T_{h c d}$, proportion of people over 65 years, $P_{065}$, and building foundation height, $H$, did not influence the LL estimates much. Since, in this study, the inputs for the LL model were chosen based on the conclusions of previous studies about their importance for the LL estimates (Table S5 in ESI), it is easier to explain why the latter four parameters are rather unimportant for this LL model. In particular, $F_{\text {compr }}$ has a small influence on the output because the inundated area is mostly in the chance zone (defined by the flood severity); $T_{h c d}$-because values chosen for this parameter are much smaller than values of other 
time characteristics in the warning and evacuation timeline (Figure 6-1 in USACE [14]); $P_{065}$-because elderly people are located in the area that is not or only slightly inundated (i.e., residential buildings in elevated parts of the locality); and $\mathrm{H}$-because the inundated area has water depths that are much higher (mainly due to the topography) than the safety buffer given by $H$.

a)

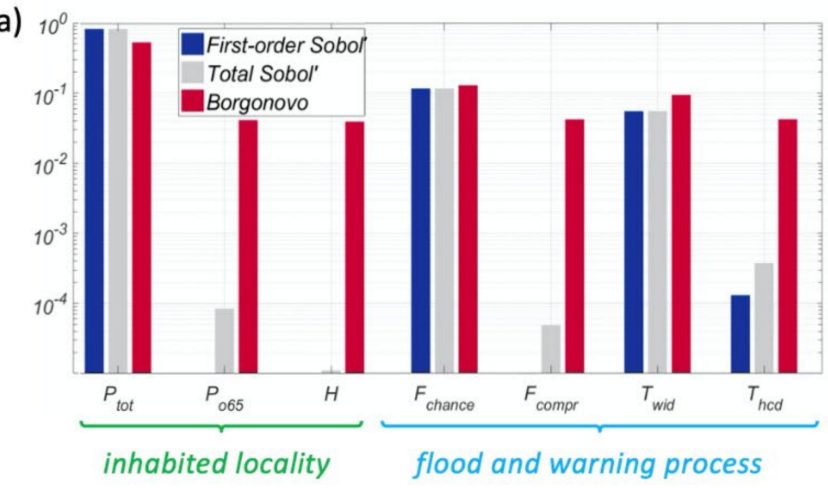

b)

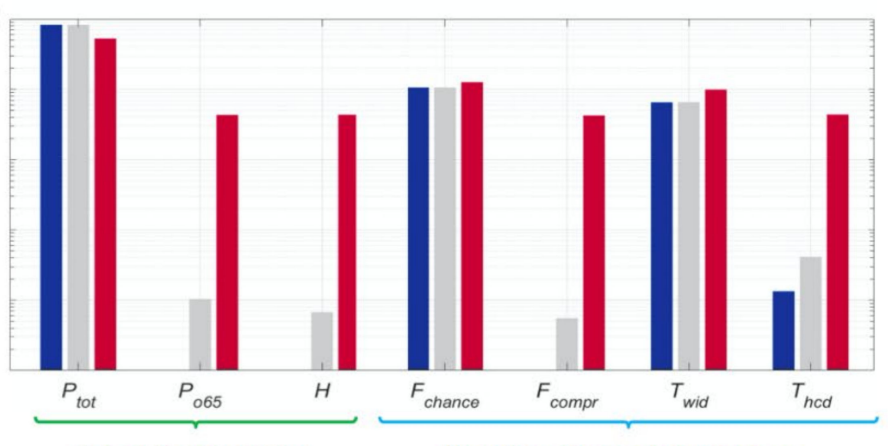

inhabited locality

flood and warning process

Figure 10. Sensitivity indices of the seven model input parameters to the LL estimates obtained in the (a) night-time and (b) daytime F50 scenarios.

Furthermore, for the majority of the model inputs, there is a negligible difference between the first-order, $S_{i}$, and total Sobol' indices, $S_{T_{i}}$, which indicates that almost all of the model variance is explained by the individual effects and there is only a small contribution to the model variance from the interaction between the assessed input parameters.

Besides computing the Sobol' indices, Borgonovo indices, $\delta_{i}$, were estimated to highlight the differences between these two sensitivity measures (Figure 10). In contrast to Sobol' indices that are variance based, Borgonovo indices are moment-independent and look at the entire input/output conditional distributions. The results reveal that both Sobol' and $\delta_{i}$ indices agree in identifying the most relevant parameters and, in general, in ranking the model inputs. Discrepancies in the indices only relate to the fact that the $\delta_{i}$ indices have higher values for the less-relevant parameters. This is because values of the $\delta_{i}$ indices indicate the sensitivity relative to the sensitivity of the most important parameter (in this study, $\left.P_{t o t}\right)$. Therefore, this ranking is a relative ranking between the model inputs.

When comparing results between night-time (Figure 10a) and daytime (Figure 10b) F50 scenarios, the ranking of the parameters remains the same; however, values of indices for $F_{c o m p r}, T_{\text {wid }}$, and $H$ are slightly higher in the daytime scenario (except $S_{F_{c o m p r}}$ ). The already influential $T_{\text {wid }}$ become more important during the day because people react much faster than at night. Therefore, an even slightly longer time interval gives more people the opportunity to react. Although $F_{\text {compr }}$ remains an unimportant input, its influence on the LL estimates slightly increases because more people may be in the compromised zone during the day. The same reason may cause an increase in values for $H$ indices since, in this zone, even slightly higher elevation of the ground floor above the ground can bring more people to safe areas.

When comparing results among all considered scenarios (F05, F50, and F95), as shown in Table S8 in ESI, a good agreement is found for the ranking of the LL model inputs for all sensitivity measures, i.e., $P_{t o t}, F_{\text {chance }}$, and $T_{\text {wid }}$. In fact, they all have a strong influence on the LL estimates in all considered scenarios. This similarity in the sensitivity ranking indicates that the effect of the change in the values of the model inputs on the LL estimates is rather proportional. However, there are still some minor changes in the values of the sensitivity coefficients among the scenarios. For example, the total Sobol' index for $H$ is different among scenarios, being the highest for F05 with the lowest water depth and flow velocities in the inundated area. Although, the ranking is similar, the computed values for the Sobol' and $\delta_{i}$ indices are different between scenarios (Table S8 in ESI). 


\section{Conclusions}

This study developed a generic framework for uncertainty quantification in the dambreak LL estimates based on the use of HEC-LIFESim and a metamodelling approach. It was subsequently applied to a case study in Switzerland. In the first part of the study, the successful application of HEC-LIFESim to model LL due to the instantaneous break of a hypothetical dam in Switzerland was demonstrated by reflecting the variation in the LL estimates depending on the time of day and flood severity. Furthermore, the benefits of applying metamodelling for uncertainty quantification in the dam-break LL estimates were shown. A polynomial chaos expansion (PCE) was applied to approximate the computational LL model created in HEC-LIFESim with a metamodel using only 550 runs, therefore, reducing the computational cost with respect to Monte Carlo (MC) based approaches. The distributions for the model inputs were defined for the full range of values representative for the population of large Swiss arch hydropower dams and their downstream localities and propagated through the metamodel to quantify uncertainty in the LL estimates. Therefore, the calculated metamodel is generic for the considered population of dams, and it can be used to support the risk assessment and management of these dams. Finally, a GSA was performed using the PCE coefficient. The GSA results indicate that the population in the locality, fatality rate in the chance zone, and warning issuance delay contributed most to the variability of the LL estimates. Furthermore, based on the differences in the model results among the six modelled scenarios, flow quantities of the dam-break flood were also found to be influential for the LL estimates; especially, they are relevant for the flow velocity.

The application of the constructed generic metamodel can be a potential step forward from the current guidelines for assessing the consequences of a dam break implemented by the Dams Supervision Division of the Swiss Federal Office of Energy [83]. The current guidelines focus on the population at risk (PAR) due to a dam break using information about flood arrival time, water depth, and flow velocity, whereas evacuation and warning processes are not considered.

Future research should focus on further data collection to improve local data (e.g., structural stability criteria for buildings in Switzerland). This is also related to the fact that the parameters, such as warning issuance delay or hazard communication delay, are set for the current model based on a review of previous research on this topic. Furthermore, it is currently unclear if consideration of additional LL scenarios, including progressive breach formation and/or modelling of muddy water propagation [82], and/or additional model input parameters would affect the conclusions made about the most influential parameters for the LL estimates. Therefore, it is important to further investigate these aspects in future studies.

Supplementary Materials: The following are available online at https:/ / www.mdpi.com/article/ 10.3390/w13233414/s1, Table S1. Data about land use, buildings, and demographic characteristics collected for all major ( $\geq 1000$ inhabitants) localities in the Rhone Valley; Table S2. Summary statistic for the characteristics of the inhabited localities in the Rhone Valley; Table S3. Assumptions made for the structural inventory in the loss of shelter module and for the warning and evacuation module in HEC-LIFESim to build the LL-model representation for a Swiss case scenario; Table S4. Different flood characteristics reflecting scenarios F05, F50, and F95; Table S5. Overview of the research conducted on LL modelling: discussions and results on methods for uncertainty and sensitivity analyses; also input parameters contributing most to uncertainty of the model output. Parameters considered for the uncertainty quantification in this study are given in bold; Table S6. The PCE degree and values of $\epsilon_{L O O}$ and MSE errors (fraction) for different model outputs using different sizes of the experimental design; Table S7. Comparison of the LL estimates, including their uncertainty, for the three scenarios F05, F50, and F95 and the two times 2 a.m. and 2 p.m. considered in this study calculated with the PCE and different MC samples (100, 1000, 10,000, 100,000) implemented in HEC-LIFESim; Table S8. The values of the 1st and total Sobol' indices and Borgonovo indices calculated for different model outputs in all six defined scenarios. 
Author Contributions: Conceptualization, A.K., M.S. and P.B.; methodology, A.K. and M.S.; formal analysis, A.K.; data curation, A.K.; writing-original draft preparation, A.K.; writing-review and editing, A.K., M.S. and P.B.; supervision, M.S. and P.B.; funding acquisition, M.S. and P.B. All authors have read and agreed to the published version of the manuscript.

Funding: This research project is part of the Risk Governance of Deep Geothermal and Hydro Energy (NRP70 Energy Turnaround) of the Swiss National Science Foundation (SNSF), grant No. 407040_153931. Further information on the National Research Programme can be found at www.nrp7 0.ch (accessed on 17 November 2021). Additionally, this work is supported by the Swiss Competence Center for Energy Research (SCCER) - Supply of Electricity (SoE) funded by the Swiss Innovation Agency (Innosuisse) grant No. 1155002546.

Data Availability Statement: All data are reported in the Supplementary Materials.

Acknowledgments: The authors express their sincere thanks to Bruno Sudret and Stefano Marelli (ETH Zurich, Switzerland) for providing access to the software and assisting while undertaking the research summarized here. Finally, Christopher Robinson (EAWAG Duebendorf and ETH Zurich, Switzerland) provided valuable feedback and support to this work.

Conflicts of Interest: The authors declare no conflict of interest.

\section{References}

1. Bureau of Reclamation. Reclamation Consequence Estimating Methodology, RCEM; Guidelines for Estimating Life Loss for Dam Safety Risk Analysis; U.S. Department of the Interior: Washington, DC, USA, 2014.

2. Darbre, G.R. Dam Risk Analysis; Dam Safety; Federal Office for Water and Geology: Bern, Switzerland, 1999.

3. Regan, P.E.P.J. An examination of dam failures vs. age of dams. In Proceedings of the 29th Annual USSD Conference, Nashville, TN, USA, 20-24 April 2009.

4. Bureau of Reclamation. Policy and Procedures for Dam Safety Modification Decisionmaking; Bureau of Reclamation: Denver, CO, USA, 1989; p. 302.

5. DeKay, M.L.; McClelland, G.H. Predicting loss of life in cases of dam failure and flash flood. Risk Anal. 1993, 13, 193-205. [CrossRef]

6. Graham, W.J. A Procedure for Estimating Loss of Life Caused by Dam Failure; DSO-99-06; United States Department of the Interior Bureau of Reclamation: Washington, DC, USA, 1999.

7. McClelland, D.M.; Bowles, D.S. Estimating Life Loss for Dam Safety Risk Assessment-A Review and New Approach; Institute for Dam Safety Risk Management Utah State University: Logan, UT, USA, 2002.

8. Mao, J.; Wang, S.; Ni, J.; Xi, C.; Wang, J. Management System for Dam-Break Hazard Mapping in a Complex Basin Environment. ISPRS Int. J. Geo Inf. 2017, 6, 162. [CrossRef]

9. Li, W.; Li, Z.; Ge, W.; Wu, S. Risk Evaluation Model of Life Loss Caused by Dam-break Flood and Its Application. Water 2019, 11, 1359. [CrossRef]

10. Yudianto, D.; Ginting, B.M.; Sanjaya, S.; Rusli, S.R.; Wicaksono, A. A Framework of Dam-Break Hazard Risk Mapping for a Data-Sparse Region in Indonesia. ISPRS Int. J. Geo Inf. 2021, 10, 110. [CrossRef]

11. Jonkman, S.N.; Vrijling, J.K. Loss of life due to floods. J. Flood Risk Manag. 2008, 1, 43-56. [CrossRef]

12. British Columbia, H. Life Safety Model System V1.0, Guidelines, Procedures, Calibration and Support Manual; British Columbia Hydro: Vancouver, BC, USA, 2006.

13. Lumbroso, D.; Davison, M. Use of an agent-based model and Monte Carlo analysis to estimate the effectiveness of emergency management interventions to reduce loss of life during extreme floods. J. Flood Risk Manag. 2018, 11, S419-S433. [CrossRef]

14. USACE. HEC-LifeSim. Life Loss Estimation. User's Manual. Version 1.0; U.S. Army Corps of Engineers, Hydrologic Engineering Center: Davis, CA, USA, 2017.

15. Bowles, D.S.; Aboelata, M. Evacuation and life-loss estimation model for natural and dam break floods. In Extreme Hydrological Events: New Concepts for Security; Vasiliev, O.F., Ed.; Springer: New York, NY, USA, 2007; Volume 78, pp. $363-383$.

16. Swisstopo. SWISSIMAGE $25 \mathrm{~cm}$. Available online: https://www.swisstopo.admin.ch/en/geodata/images/ortho/swissimage25. html (accessed on 11 January 2019).

17. Swisstopo. swissALTI3D. 2018. Available online: https://www.swisstopo.admin.ch/en/geodata/height/alti3d.html (accessed on 11 January 2019).

18. Hartford, D.; Baecher, G. Risk and Uncertainty in Dam Safety; Thomas Telford Publishing: London, UK, 2004.

19. Qi, W.; Zhang, C.; Fu, G.; Sweetapple, C.; Liu, Y. Impact of robustness of hydrological model parameters on flood prediction uncertainty. J. Flood Risk Manag. 2018, 12, e12488. [CrossRef]

20. Graham, W.J. A comparison of methods for estimating loss of life from dam failure. In Proceedings of the Managing Our Water Retention Systems, 29th Annual USSD Conference, Nashville, TN, USA, 20-24 April 2009.

21. Lee, J.S. Uncertainties in the Predicted Number of Life Loss due to the Dam Breach Floods. KSCE J. Civ. Eng. 2003, 7, 81-91. [CrossRef] 
22. El Bilali, A.; Taleb, A.; Boutahri, I. Application of HEC-RAS and HEC-LifeSim models for flood risk assessment. J. Appl. Water Eng. Res. 2021, 1-16. [CrossRef]

23. Aboelata, M.; Bowles, D.S. LIFESim: A tool for estimating and reducing life-loss resulting from dam and levee failures. In Proceedings of the Association of State Dam Safety Officials "Dam Safety 2008" Conference, Indian Wells, CA, USA, 7-11 September 2008.

24. Aboelata, M.; Bowles, D.S.; McClelland, D.M. A Model for estimating dam failure life loss. In Proceedings of the ANCOLD 2003 Conference on Dams, The Australian Committee on Large Dams Risk Workshop, Launceston, TAS, Australia, October 2003.

25. Byrne, M.D. How many times should a stochastic model be run? An approach based on confidence intervals. In Proceedings of the 12th International Conference on Cognitive Modeling, Ottawa, ON, Canada, 11-14 July 2013.

26. Le Gratiet, L.; Marelli, S.; Sudret, B. Metamodel-based sensitivity analysis: Polynomial chaos expansions and gaussian processes. In Handbook of Uncertainty Quantification; Ghanem, R., Higdon, D., Owhadi, H., Eds.; Springer International Publishing: Cham, Switzerland, 2017; pp. 1289-1325.

27. Sudret, B. Uncertainty Propagation and Sensitivity Analysis in Mechanical Models: Contributions to Structural Reliability and Stochastic Spectral Methods; Habilitation àdiriger des Recherches; Université Blaise Pascal: Clermont-Ferrand, France, 2007.

28. De Rocquigny, E.; Devictor, N.; Tarantola, S. Uncertainty in Industrial Practice-A Guide to Quantitative Uncertainty Management; John Wiley \& Sons: Hoboken, NJ, USA, 2008.

29. Xiu, D.; Karniadakis, G. The Wiener-Askey polynomial chaos for stochastic equations. SIAM J. Sci. Comput. 2002, 24, 619-644. [CrossRef]

30. Aboelata, M.A.; Bowles, D.S. LIFESim: A Model for Estimating Dam Failure Life Loss; Report to Institute for Water Resources, US Army Corps of Engineers and Australian National Committee on Large Dams; Institute for Dam Safety Risk Management, Utah State University: Logan, UT, USA, 2005.

31. Saltelli, A.; Chan, K.; Scott, E.M. Sensitivity Analysis: Wiley Series in Probability and Statistics; John Wiley: Chichester, UK, 2000.

32. Mokhtari, A.; Frey, H.C. Sensitivity analysis of a two-dimensional probabilistic risk assessment model using analysis of variance. Risk Anal. 2005, 25, 1511-1529. [CrossRef]

33. Lang, M.; Rehan, B.M.; Hall, J.W.; Klijn, F.; Samuels, P. Uncertainty and sensitivity analysis of flood risk management decisions based on stationary and nonstationary model choices. E3S Web Conf. 2016, 7, 20003. [CrossRef]

34. Kalinina, A.; Spada, M.; Vetsch, D.; Marelli, S.; Whealton, C.; Burgherr, P.; Sudret, B. Metamodeling for Uncertainty Quantification of a Flood Wave Model for Concrete Dam Breaks. Energies 2020, 13, 3685. [CrossRef]

35. Saltelli, A.; Ratto, M.; Andres, T.; Campolongo, F.; Cariboni, J.; Galetti, D.; Saisana, M.; Tarantola, S. Global Sensitivity Analysis: The Primer; Wiley: Hoboken, NJ, USA, 2008.

36. Delenne, C.; Cappelaere, B.; Guinot, V. Uncertainty analysis of river flooding and dam failure risks using local sensitivity computations. Reliab. Eng. Syst. Saf. 2012, 107, 171-183. [CrossRef]

37. Sobol, I.M. Global sensitivity indices for nonlinear mathematical models and their Monte Carlo estimates. Math. Comput. Simul. 2001, 55, 271-280. [CrossRef]

38. Frey, H.C.; Patil, S.R. Identification and review of sensitivity analysis methods. Risk Anal. 2002, 22, 553-578. [CrossRef]

39. Tene, M.; Stuparu, D.E.; Kurowicka, D.; El Serafy, G.Y. A copula-based sensitivity analysis method and its application to a North Sea sediment transport model. Environ. Model. Softw. 2018, 104, 1-12. [CrossRef]

40. Borgonovo, E. A new uncertainty importance measure. Reliab. Eng. Syst. Saf. 2007, 92, 771-784. [CrossRef]

41. Iooss, B.; Lemaître, P. A review on global sensitivity analysis methods. In Uncertainty Management in Simulation-Optimization of Complex Systems: Algorithms and Applications; Dellino, G., Meloni, C., Eds.; Springer US: Boston, MA, USA, $2015 ;$ pp. 101-122.

42. Zhang, L.; Peng, M.; Chang, D.; Xu, Y. Dam Failure Mechanisms and Risk Assessment, 1st ed.; Jon Wiley \& Sons Singapore Pte. Ltd.: Singapore, 2016.

43. ESRI. ESRI Products, Software, and Services; Environmental Systems Research Institute: Redlands, CA, USA, 2017.

44. Bowles, D.S. Life loss estimation for RAMCAP, Appendix D. In Conventional Dams and Navigation Locks, Sector-Specific Guidance (SSG), Risk Analysis and Management for Critical Asset Protection (RAMCAP) Phase III for Dams, Locks and Levees; CISA: Arlington, VA, USA, 2007.

45. McClelland, D.M.; Bowles, D. Estimating life loss for dam safety and risk assessment: Lessons from case histories. In Proceedings of the 2000 Annual USCOLD Conference, U.S. Society on Dams, Denver, CO, USA, 5-8 August 2000.

46. Branch, M.C. Common characteristics of new towns. Cities 1983, 1, 146-149. [CrossRef]

47. Chen, Y.J.; Matsuoka, R.H.; Liang, T.M. Urban form, building characteristics, and residential electricity consumption: A case study in Tainan City. Environ. Plan. B Urban Anal. City Sci. 2017, 45, 933-952. [CrossRef]

48. SFSO. Regionalporträts 2017: Kennzahlen aller Gemeinden; je-d-21.03.01; Swiss Federal Statistical Office: Bern, Switzerland, 2017.

49. SFSO. Eidgenössisches Gebäude- und Wohnungsregister. Version 3.7.; The Federal Department of Home Affairs: Neuchâtel, Switzerland, 2018.

50. FEI. Development of Rescue Actions Based on Dam-Break Flood Analysis, RESCDAM; Finnish Environmental Insititute: Helsinki, Finland, 2001.

51. World Bank. Doing Business 2019. Training for Reform; World Bank: Washington, DC, USA, 2019.

52. USACE. HEC-RAS 5.0.3; U.S. Army Corps of Engineers, Hydrologic Engineering Center: Davis, CA, USA, 2017. 
53. SFSO. Ständige und Nichtständige Wohnbevölkerung Nach Institutionellen Gliederungen, Geburtsort und Staatsangehörigkeit; (STAT-TAB); Swiss Federal Statistical Office: Bern, Switzerland, 2017. (In German)

54. WRFA. Water Retaining Facilities Act; WRFA: Jamestown, NY, USA, 2013.

55. SFOE. Directive on the Safety of Water Retaining Facilities, Part D: Commissioning and Operation; Swiss Federal Office of Energy: Bern, Switzerland, 2015.

56. Public Safety Department of the Municipality of Brig-Glis. Notfallinfo. Available online: https://www.brig-glis.ch/sicherheit/ (accessed on 8 October 2018).

57. OpenStreetMap. Open Data Commons Open Database License. 2018. Available online: https://opendatacommons.org/licenses / $\mathrm{odbl} /$ (accessed on 8 October 2018).

58. Kalinina, A.; Spada, M.; Burgherr, P. Alternative life-loss rates for failures of large concrete and masonry dams in mountain regions of OECD countries. In Proceedings of the Safety and Reliability of Complex Engineered Systems: ESREL, Trondheim, Norway, 17-21 June 2018.

59. Zellnerr, A.; Highfiled, R. Calculation of Maximum Entropy Distributions and Approximation of Marginal Posterior Distributions. J. Econom. 1988, 37, 195-209. [CrossRef]

60. Lingam, M.; Comisso, L. A maximum entropy principle for inferring the distribution of 3D plasmoids. Phys. Plasmas 2018, 25, 012114. [CrossRef]

61. De Martino, A.; De Martino, D. An introduction to the maximum entropy approach and its application to inference problems in biology. Heliyon 2018, 4, e00596. [CrossRef] [PubMed]

62. Bhat, H.S.; Kumar, N. On the Derivation of the Bayesian Information Criterion; School of Natural Sciences, University of California: Oakland, CA, USA, 2010.

63. Akaike, H. A new look at the statistical model identification. IEEE Trans. Autom. Control 1974, 19, 716-723. [CrossRef]

64. Limpert, E.; Stahel, W.A.; Abbt, M. Log-normal Distributions across the Sciences: Keys and Clues. BioScience 2001, 51, 341-352. [CrossRef]

65. Matz, A.W. Maximum Likelihood Parameter Estimation for the Quartic Exponential Distribution. Technometrics 1978, 20, 475-484. [CrossRef]

66. Ghanem, R.; Spanos, P. Stochastic Finite Elements—A Spectral Approach; Springer: New York, NY, USA, 1991.

67. McKay, M.D.; Beckman, R.J.; Conover, W.J. A comparison of three methods for selecting values of input variables in the analysis of output from a computer code. Technometrics 1979, 2, 239-245.

68. Efron, B.; Hastie, T.; Johnstone, I.; Tibshirani, R. Least angle regression. Ann. Stat. 2004, 32, 407-499. [CrossRef]

69. Marelli, S.; Sudret, B. UQLab: A framework for uncertainty quantification in Matlab 257 Vulnerability, Uncertainty, and Risk. In Proceedings of the 2nd International Conference on Vulnerability, Risk Analysis and Management (ICVRAM2014), Liverpool, UK, 13-16 July 2014; pp. 2554-2563.

70. Marelli, S.; Sudret, B. UQLab User Manual_Polynomial Chaos Expansions; Technical Report; Chair of Risk, Safety \& Uncertainty Quantification, ETH: Zürich, Switzerland, 2017.

71. Blatman, G.; Sudret, B. Adaptive sparse polynomial chaos expansion based on Least Angle Regression. J. Comput. Phys. 2011, 230, 2345-2367. [CrossRef]

72. Blatman, G.; Sudret, B. An adaptive algorithm to build up sparse polynomial chaos expansions for stochastic finite element analysis. Probabilistic Eng. Mech. 2010, 25, 183-197. [CrossRef]

73. Marelli, S.; Lamas, C.; Sudret, B.; Konakli, K.; Mylonas, C. UQLab User Manual—Sensitivity Analysis; Chair of Risk, Safety \& Uncertainty Quantification, ETH: Zürich, Switzerland, 2018.

74. Brevault, L.; Balesdent, M.; Berend, N.; Le Riche, R. Comparison of different global sensitivity analysis methods for aerospace vehicle optimal design. In Proceedings of the 10th World Congress on Structural and Multidisciplinary Optimization, Orlando FL, USA, 19-24 May 2013.

75. Zhang, X.-Y.; Trame, M.N.; Lesko, L.J.; Schmidt, S. Sobol Sensitivity Analysis: A Tool to Guide the Development and Evaluation of systems Pharmacology Models. CPT Pharmacomet. Syst. Pharmacol. 2015, 4, 69-79. [CrossRef]

76. Sobol, I. Sensitivity estimates for nonlinear mathematical models. Math. Comput. Model. 1993, 1, 407-414.

77. Sudret, B. Global sensitivity analysis using polynomial chaos expansions. Reliab. Eng. Syst. Saf. 2008, 93, 964-979. [CrossRef]

78. Baecher, G.B. Uncertainty in dam safety risk analysis. GEORISK 2016, 10, 92-108. [CrossRef]

79. Graham, W.J. A Procedure for Estimating Loss of Life Caused by Dam Failure; Sedimentation \& River Hydraulics: Denver, CO, USA, 1999.

80. Wang, C.; Zhand, S.; Tan, Y.; Pan, F.; Yan, L. Life Loss Estimation Based on Dam-Break Flood Uncertainties and Lack of Information in Mountainous Regions of Western China. Trans. Tianjin Univ. 2017, 23, 370-379. [CrossRef]

81. Hirschberg, S.; Spiekerman, G.; Dones, R. Severe Accidents in the Energy Sector, 1st ed.; PSI Report 98-16; Paul Scherrer Institut: Villigen PSI, Switzerland, 1998.

82. Darbre, G.R. Dam breach in the context of dam safety legislation. In Proceedings of the SwissCOD/VAW Workshop on Dam Breach Analysis, Bern, Switzerland, 26 April 2017.

83. SFOE. Methodik zur Bestimmung der Anzahl Gefährdeter Personen (People at Risk PAR) zur Abschätzung der Hohen Gefahr (Version 1.0); Swiss Federal Office of Energy, Sektion Aufsicht Talsperren: Bern, Switzerland, 2017. (In German) 